\title{
Stellar populations in a standard ISOGAL field in the Galactic disc ${ }^{\star} \star \star$
}

\author{
S. Ganesh ${ }^{1,2}$, A. Omont ${ }^{1}$, U. C. Joshi ${ }^{2}$, K. S. Baliyan ${ }^{2}$, M. Schultheis ${ }^{3,1}$, F. Schuller ${ }^{4,1}$, and G. Simon ${ }^{5}$ \\ 1 Institut d'Astrophysique de Paris, CNRS and Université Paris 6, 98bis boulevard Arago, 75014 Paris, France \\ e-mail: shashi@iap.fr \\ 2 Physical Research Laboratory, Navrangpura, Ahmedabad-380 009, India \\ e-mail: shashi@prl.res.in \\ Observatoire de Besançon, Besançon, France \\ 4 Max-Planck-Institut fur Radioastronomie, Auf dem Hugel 69, 53121 Bonn, Germany \\ 5 GEPI, UMS-CNRS 2201, Observatoire de Paris, France
}

Received 28 February 2008 / Accepted 3 September 2008

ABSTRACT

\begin{abstract}
Aims. We identify the stellar populations (mostly red giants and young stars) detected in the ISOGAL survey at 7 and $15 \mu \mathrm{m}$ towards a field (LN45) in the direction $\ell=-45, b=0.0$.

Methods. The sources detected in the survey of the Galactic plane by the Infrared Space Observatory were characterised based on colour-colour and colour-magnitude diagrams. We combine the ISOGAL catalogue with the data from surveys such as 2MASS and GLIMPSE. Interstellar extinction and distance were estimated using the red clump stars detected by 2MASS in combination with the isochrones for the AGB/RGB branch. Absolute magnitudes were thus derived and the stellar populations identified from their absolute magnitudes and their infrared excess.

Results. A standard approach to analysing the ISOGAL disc observations has been established. We identify several hundred RGB/AGB stars and 22 candidate young stellar objects in the direction of this field in an area of $0.16 \mathrm{deg}^{2}$. An overdensity of stellar sources is found at distances corresponding to the distance of the Scutum-Crux spiral arm. In addition, we determined mass-loss rates of AGB-stars using dust radiative transfer models from the literature.
\end{abstract}

Key words. infrared: stars - Galaxy: stellar content - ISM: dust, extinction

\section{Introduction}

ISOGAL survey data at 7 and $15 \mu \mathrm{m}$ of about $16 \mathrm{deg}^{2}$ in selected fields of the inner Galactic disc have allowed detection of about $1 \times 10^{5}$ point sources down to $\sim 10 \mathrm{mJy}$ at $15 \mu \mathrm{m}$ and $7 \mu \mathrm{m}$. In conjunction with ground-based near-IR surveys (DENIS, 2MASS), they offer the possibility of investigating the different populations of infrared stars in the Galactic disc up to $15 \mu \mathrm{m}$. The technical characteristics of the five wavelength ISOGAL-DENIS point source catalogue are presented and discussed in detail by Schuller et al. (2003), while Omont et al. (2003) reviewed the scientific capabilities and the main outcome of ISOGAL.

The best-detected stellar class is that of AGB stars which are almost completely detected above the RGB tip at least at $7 \mu \mathrm{m}$ up to the Galactic centre (Glass et al. 1999; Omont et al. 1999). The infrared colour $\left(K_{\mathrm{S}}-[15]\right)_{0}$ is a very good measure of their mass-loss rate (Ojha et al. 2003). The mass-loss

\footnotetext{
* Based on observations with ISO, an ESA project with instruments funded by ESA Member States (especially the PI countries: France, Germany, the Netherlands and the United Kingdom) and with the participation of ISAS and NASA.

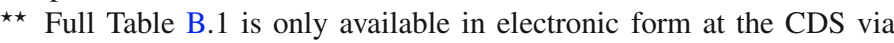
anonymous ftp to cdsarc.u-strasbg.fr (130.79.128.5) or via http://cdsweb.u-strasbg.fr/cgi-bin/qcat?J/A+A/493/785
}

rate can also be derived from the pure ISOGAL colour [7]-[15], which is practically independent of extinction. Combined with variability data from MACHO (Alcock et al. 1997) or EROS (Palanque-Delabrouille et al. 1998), ISOGAL data of bulge fields have shown that practically all sources detected at $15 \mu \mathrm{m}$ are long-period variables with interesting correlations between the mass-loss rate traced by the $15 \mu \mathrm{m}$ excess and variability intensity and period (Alard et al. 2001). The $15 \mu \mathrm{m}$ data from the fields observed $\left(0.29 \mathrm{deg}^{2}\right.$ in total $)$ in the Galactic bulge $\left(|b| \geq 1^{\circ}\right)$ have been used by Ojha et al. (2003) to infer global properties of the mass returned to the interstellar medium by AGB stars in the bulge. However, similar work has not yet been carried out in the disc ISOGAL fields because of the difficulty in properly estimating distances along the line of sight.

Red giants are by far the most numerous population of luminous bright stars in the near and mid-infrared. They are detectable through out the Galaxy with modern surveys such as 2MASS, DENIS, GLIMPSE, ISOGAL, and MSX. The reddening of various infrared colours may be used to trace the interstellar extinction, $A_{\mathrm{V}}$. However, the near-IR colours $J-K_{\mathrm{S}}$ or $H-K_{\mathrm{S}}$ are generally the most useful because of greater sensitivity to extinction with $A_{J}-A_{K \mathrm{~s}} \sim 0.17 A_{\mathrm{V}}$ and $A_{\mathrm{H}}-A_{K \mathrm{~s}} \sim 0.06 A_{\mathrm{V}}$ (Glass 1999). Such methods have been used for systematic studies of the extinction in large areas from DENIS (Schultheis et al. 1999) and 2MASS (Dutra et al. 2003), and also for modelling of 
Galactic stellar populations from these surveys (Marshall et al. 2006).

When the luminosities of the red giants are known, e.g. those of the "red clump" or the RGB tip, one may infer both extinction and the distance from colour-magnitude diagrams (CMD) such as $J$ vs. $J-K_{\mathrm{S}}$. The numerous giants of the red clump are known to be, by far, the best way to make $3 \mathrm{D}$ estimates of the extinction along Galactic lines of sight by determining distance scales from their well-defined luminosities and intrinsic colours. López-Corredoira et al. (2002), and Drimmel et al. (2003), and Indebetouw et al. (2005) have exploited the red clump stars of DENIS and 2MASS to explore the extinction along various lines of sight.

The $6-8 \mu \mathrm{m}$ range is not as good an indicator of AGB mass loss as $15 \mu \mathrm{m}$; however, the sensitivity of ISOGAL at $7 \mu \mathrm{m}$ allows detection of less luminous giants below the RGB tip at the distance of the bulge (Glass et al. 1999). The large number of such stars, $\sim 10^{5}$, detected by ISOGAL in the Galactic disc and inner bulge may also be used to study the mid-infrared extinction law at $7 \mu \mathrm{m}$ by comparing $K_{\mathrm{S}}-$ [7] with $J-K_{\mathrm{S}}$. Jiang et al. (2003) have used this approach along one line of sight. This method may even be extended to deriving extinction at $15 \mu \mathrm{m}$ from the ratio $K_{\mathrm{S}}-[15] / J-K_{\mathrm{S}}$. It has recently been applied to all the exploitable ISOGAL lines of sight (more than 120 directions at both wavelengths of ISO) in the Galactic disc and inner bulge (Jiang et al. 2006).

Young stars with dusty discs or cocoons are the other class of objects to be addressed using ISOGAL's ability to detect $15 \mu \mathrm{m}$ excess. Felli et al. (2002) have thus identified 715 candidate young stars from relatively bright ISOGAL sources with a very red [7]-[15] colour. Schuller (2002) has proposed another criteria for identifying such luminous young stellar objects based on the non-point source like behaviour of the $15 \mu \mathrm{m}$ emission. However, various situations have limited the full exploitation of ISOGAL for young-star studies; e.g. lack of complementary data at longer or shorter wavelengths, making it difficult to have a good diagnostic of the nature of the objects, their luminosity, and mass; lack of angular resolution that may preclude deblending of nearby sources; limited quality of the data, especially in the regions of active star formation with high diffuse infrared background.

Other infrared surveys, IRAS and MSX (Price et al. 2001), have covered the complete Galactic disc, including bands at longer wavelengths, but with a very limited sensitivity, especially in the range $12-20 \mu \mathrm{m}$ where ISOGAL is three or four magnitudes deeper than MSX. However, the much increased panoramic capabilities of the Spitzer Space Observatory have now made available much deeper data in the four IRAC bands $(3.6,4.5,5.8$, and $8.0 \mu \mathrm{m})$ in the main part of the whole Galactic disc from the GLIMPSE Spitzer Legacy Project (Benjamin et al. 2003, 2005), extended to the whole inner disc with GLIMPSE II. GLIMPSE is about one order of magnitude deeper than ISOGAL in the range $6-8 \mu \mathrm{m}$, and it has a better angular resolution, but it lacks extension at longer wavelengths as provided by ISOGAL at $15 \mu \mathrm{m}$. We note that the MIPSGAL project with Spitzer will provide longer wavelength (MIPS bands at $24 \mu \mathrm{m}$ and $70 \mu \mathrm{m}$ ) coverage in the near future (Carey et al. 2005).

The main purpose of the present paper is to begin a reassessment of ISOGAL data in conjunction with the availability of GLIMPSE data. We have chosen a standard ISOGAL field with good quality data that covers a relatively large area and latitude range, allowing a valuable statistical study. We made a complete analysis of the ISOGAL data and validated their quality using the GLIMPSE data. We discuss their various science outputs, especially at $15 \mu \mathrm{m}$, including complementary information in the line of sight, especially from GLIMPSE/2MASS and CO millimetre observations. Our main goal in such a case study is to validate general methods for a subsequent complete exploitation of $15 \mu \mathrm{m}$ data in all ISOGAL fields, complemented by near-IR and GLIMPSE data, especially for systematic studies of AGB stars and their mass loss and dusty young stars of intermediate mass.

The paper is organised as follows. Section 2 recalls the general properties of ISOGAL data and describes the associations of ISOGAL point sources in this field with GLIMPSE, 2MASS, and MSX sources. Section 3 discusses corresponding statistics and the validation of ISOGAL quality using GLIMPSE data. Section 4 is devoted to interstellar extinction in this direction, the mid-IR extinction law and stellar dereddening, and comparison with $\mathrm{CO}$ emission across the field, in order to infer a rough three-dimensional picture of extinction and source distribution. Section 5 deals with the nature of the sources: the AGB population, its mass-loss, luminosity function and relation with the RGB population, identification of the relatively few young stars in this direction and their properties, and relationship with various other indicators of star formation.

\section{The data set}

\subsection{ISOGAL observations and data reduction}

The details of the ISOGAL observations with ISOCAM (Cesarsky et al. 1996) and the general processing of the data are described in Schuller et al. (2003). The reduction of the data was performed first using the standard "CIA" package of ISOCAM pipeline (Ott et al. 1999), with post-processing specific to ISOGAL data including (i) simulation of the time behaviour of the pixels of the ISOCAM detectors; (ii) PSF source extraction optimised for crowded fields; (iii) removal of residual source-saturation artefacts; (iv) specific photometric calibration; (v) 7-15 $\mu \mathrm{m}$ band merging etc. (Schuller et al. 2003).

For the present case study, we selected an ISOGAL field in the disc, with a large area $\left(0.16 \mathrm{deg}^{2}\right), \mathrm{LN} 45$, towards longitude $\ell=-45^{\circ}$, covering the latitude range $|b|<0.5^{\circ}$. The LN45 field is designated as FC-04496+0000 in the ISOGAL PSC (Schuller et al. 2003), where FC represents fields observed in both $7 \mu \mathrm{m}$ and $15 \mu \mathrm{m}$ and the next digits represent the galactic longitude and latitude of the centre of the field. The data are of good quality with repeated observations (two observations at $15 \mu \mathrm{m}$ ) for quality verifications. As shown in Fig. 1, this is in a direction tangential to the Scutum-Crux arm, and it also crosses the SagittariusCarina arm (Russeil 2003). A colour-composite image resulting from the ISOGAL observations is shown in Fig. 2. The young stellar object candidates (YSO, see Sect. 5.2) are marked in this figure. The observational parameters are reported in Table 1. A preliminary analysis of the same field, observed as part of the science verifications of ISOCAM, has been presented by Perault et al. (1996).

Broad band ISOCAM filters at $7 \mu \mathrm{m}(L W 2)$ and $15 \mu \mathrm{m}$ ( $L W 3$, Table 1) were used to increase the sensitivity as in most ISOGAL observations. Conversion factors from flux-density to Vega magnitudes are:

$[7]=12.38-2.5 \log F_{L W 2}(\mathrm{mJy})$

$[15]=10.79-2.5 \log F_{L W 3}(\mathrm{mJy})$. 


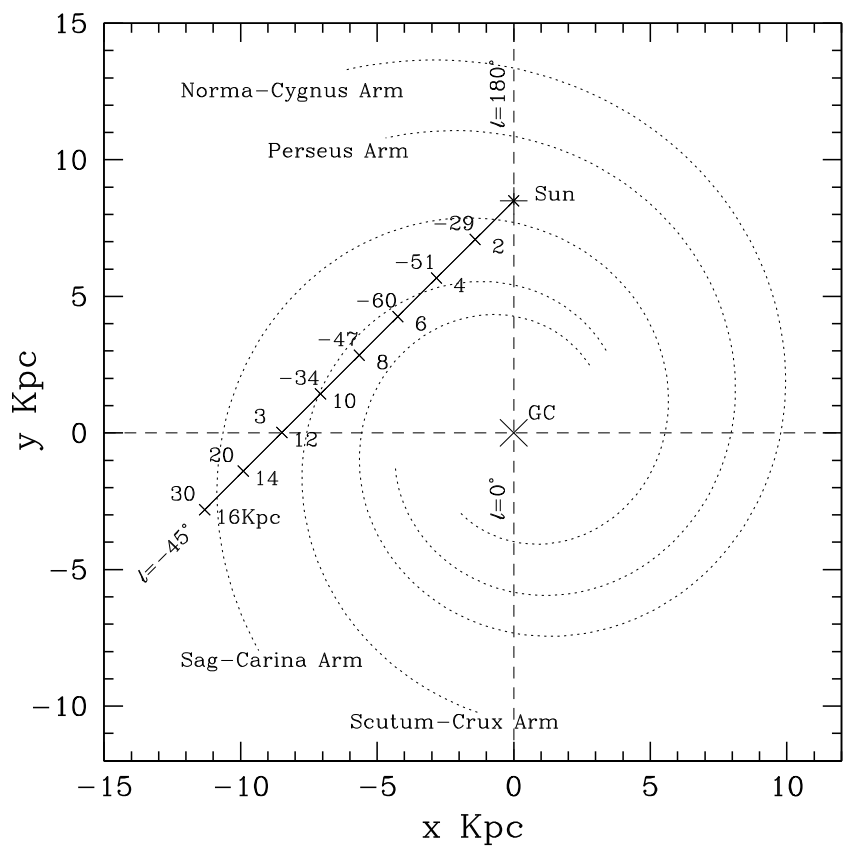

Fig. 1. The LN45 direction is shown as a solid line overlaid on the spiral arms (dotted curves) from Russeil (2003). Distances are marked every $2 \mathrm{kpc}$ below the solid line. Numbers above the solid line are the kinematic velocities in $\mathrm{km} \mathrm{s}^{-1}$ of the CO clouds (Lang 1999) at those distances.

The standard ISOGAL-DENIS catalogue for this field has been constructed using the ISO observations 24901257 and 60600458 in $L W 2$ and $L W 3$, respectively. For the source identification and band-merging procedures used, we refer to Schuller et al. (2003) and Omont et al. (2003). The number of sources extracted are 699 and 352 respectively in the $L W 2$ and $L W 3$ bands within the limiting magnitudes 9.87 and 8.62 , corresponding to flux limits of about $10 \mathrm{mJy}$ and $7 \mathrm{mJy}$, respectively. In the ISOGALDENIS point source catalogue (PSC), there are thus a total of 746 ISOCAM sources, out of which 305 objects are detected in both the $L W 2$ and $L W 3$ bands, 394 objects detected only in $L W 2$ and 47 objects detected only in the $L W 3$ band. Among the $305 L W 2-L W 3$ associated sources, 289 sources have good association quality flags ( 3 or 4 ), and 16 sources have doubtful associations with quality flag 2 (Schuller et al. 2003). With an association radius of 5.4" and a one-sigma distance uncertainty of $1.7^{\prime \prime}$, the upper limit to the number of chance associations between $L W 2-L W 3$ is less than 1 ( $0.3 \%$ of 305 common sources).

The dedicated DENIS data for this field, as published in version 1 of the ISOGAL-DENIS PSC catalogue, are extracted from a series of observations in 1996 and 2000. We complemented this set with additional DENIS data from the regular DENIS observations (strips) for the whole field. The identification procedures employed are the same as discussed in detail by Schuller et al. (2003).

\subsection{Cross identification with other surveys}

Sources in the ISOGAL-DENIS catalogue were cross-identified with corresponding sources in the other large-scale surveys 2MASS, GLIMPSE, and MSX. The procedure adopted is discussed below. At all stages the process was tailored to reduce the possibility of chance associations.

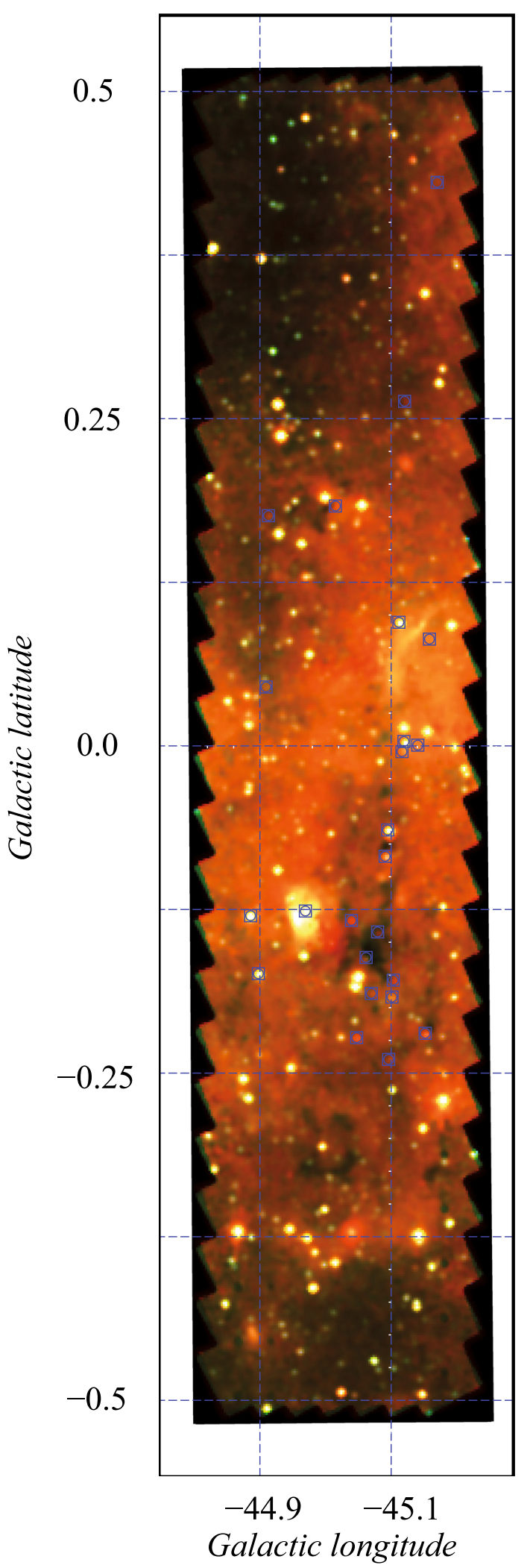

Fig. 2. False colour composite of the LN45 field with the $7 \mu$ m images being set in the green and blue channels and the $15 \mu \mathrm{m}$ represented in the red channel to make up an RGB image. The individual channels have been scaled differently to bring out the extended emission: red colour represents the $15 \mu \mathrm{m}$ image with histogram equalisation while the green and blue colour represent the $7 \mu$ m image linearly scaled between two different limits. Galactic coordinate grid is overlaid. Images were Gaussian-smoothed with a 2 pixel radius prior to composition. Positions of the YSO candidates are marked with blue square symbols. 
Table 1. Details of the ISOGAL observations discussed in the present paper.

\begin{tabular}{ccclcccccc}
\hline \hline$\ell$ & $b$ & TDT & Observation date & ISO-ID & $\delta \ell / 2$ & $\delta b / 2$ & RA(2000) & Dec(2000) & Filter \\
\hline 315.042 & +00.000 & 24901257 & 23 July 1996 & LN4500A & 0.105 & 0.507 & 218.067 & -60.477 & $L W 2$ \\
315.040 & +00.004 & 30601587 & 18 September 1996 & LN4500A & 0.125 & 0.500 & 218.044 & -60.481 & $L W 3$ \\
315.040 & +00.001 & 60600458 & 14 July 1997 & 3N45P0 & 0.100 & 0.502 & 218.062 & -60.475 & $L W 3$ \\
\hline
\end{tabular}

Notes: i) the ISOCAM filter bandwidths (half-maximum transmission points), are 5.0-8.5 $\mu \mathrm{m}$ for $L W 2$ and $12.0-18.0 \mu \mathrm{m}$ for $L W 3$ see (Blommaert et al. 2003); ii) $\delta \ell / 2$ and $\delta b / 2$ are the dimensions in degree of the rectangular field retained for this study within the observed field, to avoid edge effects on the quality of data and associations (Schuller et al. 2003); iii) observations are with 6" pixels.

Table 2. The different association flags and number statistics.

\begin{tabular}{crl}
\hline \hline Flag & Number of sources & Remarks \\
\hline 9 & 3 & ISOGAL sources saturated in GLIMPSE IRAC4 \\
8 & 3 & ISOGAL sources of intermediate brightness missing in GLIMPSE \\
7 & 2 & ISOGAL sources of intermediate brightness with GLIMPSE counterpart at $r>3.8$ and $r<4.5$ \\
5 & 355 & Very secure association between ISOGAL and GLIMPSE \\
4 & 188 & Secure association between ISOGAL and GLIMPSE \\
3 & 80 & Probable association \\
2 & 41 & Possible association \\
1 & 9 & Dubious association, probably real but blended sources \\
0 & 65 & Rejected sources \\
\hline Total & 746 & Number of sources in the ISOGAL-DENIS PSC version 1 (Schuller et al. 2003) \\
\hline
\end{tabular}

\subsubsection{Association between ISOGAL-DENIS and GLIMPSE-2MASS}

Version 2 of the GLIMPSE catalogue ${ }^{1}$ provides the 2 MASS photometry for the GLIMPSE counterparts. The total number of sources extracted in the IRAC4 band of the GLIMPSE-2MASS catalogue is 4601 . We note that sources saturated in GLIMPSE but not saturated in 2MASS are not included in the version 2 of the GLIMPSE-2MASS catalogue. The saturation limit in GLIMPSE IRAC4 band is 4 mag.

The Spitzer pointing accuracy is better than $1^{\prime \prime}$ (Werner et al. 2004; McCallon et al. 2007). The ISOGAL/DENIS catalogue PSC has its astrometry derived from that of the DENIS $I$ band, which in turn is based on the USNO catalogue. Therefore the positional uncertainty in the case of the ISOGAL PSC is also about $1^{\prime \prime}$.

The sources in the ISOGAL-DENIS catalogue were crossidentified with the GLIMPSE-2MASS catalogue using search radii of $3.8^{\prime \prime}$. To have a probability of a chance association below $10 \%$, the search radius, $r_{\mathrm{s}}$, was computed from the requirement that $n \pi r_{\mathrm{s}}^{2}=0.1$, where $n$ is the source density of the GLIMPSE2MASS catalogue. Here, $n=28756$ sources per sq. deg giving $r_{\mathrm{s}}=3.8^{\prime \prime}$. The probability of false associations drops to less than $4 \%$ when one considers that $95 \%$ of the ISOGAL sources are associated to a GLIMPSE-2MASS source within 2.3". Based on the association distance and various photometric criteria, we define a set of quality flags as listed in Table 2.

The definition of the association or quality flags (based on a combination of photometry and astrometry) is described in detail in the readme file accompanying the electronic version of the LN45 catalogue and also in the online Appendix (see Sect. A) with this article. Figure 3 illustrates the flag definitions for the cases where associations exist between GLIMPSE and ISOGAL (on a scale of 1 to 5). We list the total number of sources with the various flags in our catalogue in Table 2. Sources with a flag of 0 (cases of weak detections in only one of

\footnotetext{
1 See http://data.spitzer.caltech.edu/popular/glimpse/ 20070416_enhanced_v2/source_lists/
}

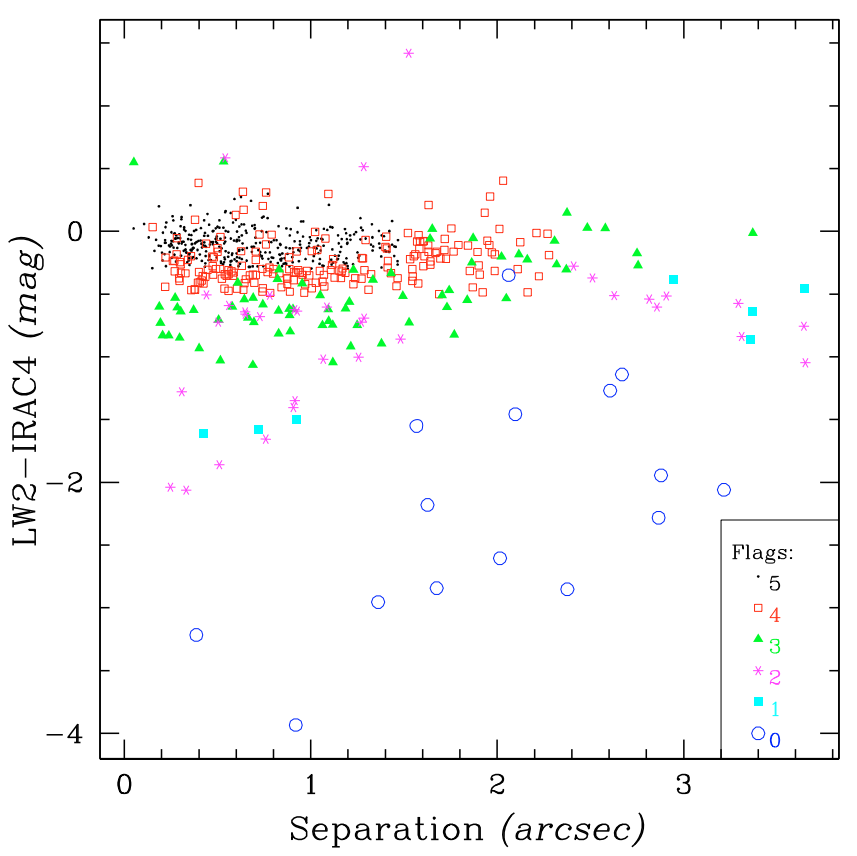

Fig. 3. The relative reliability of the sources as a function of distance of association and $7-8 \mu \mathrm{m}$ colour. This is used to define the various flags (from 0 to 5 ).

the ISO bands) are excluded from the discussion. The final catalogue for the LN45 field contains 681 sources (after excluding the flag 0 sources) out of which 8 sources do not have GLIMPSE counterparts in the IRAC4 band within $3.8^{\prime \prime}$. Three of these are cases due to saturation in the $8 \mu \mathrm{m}$ band $($ flag $=9)$. Five relatively bright sources found in the ISOGAL catalogue but not in the GLIMPSE $8 \mu \mathrm{m}$ (but below the saturation limits of GLIMPSE) are apparently extended sources or blends. There are no $8 \mu \mathrm{m}$ sources within $5^{\prime \prime}$ of the ISOGAL source in the GLIMPSE survey for three of these sources (flag $=8$ ), while the other two have GLIMPSE counterparts at 4.2" (flag =7). 


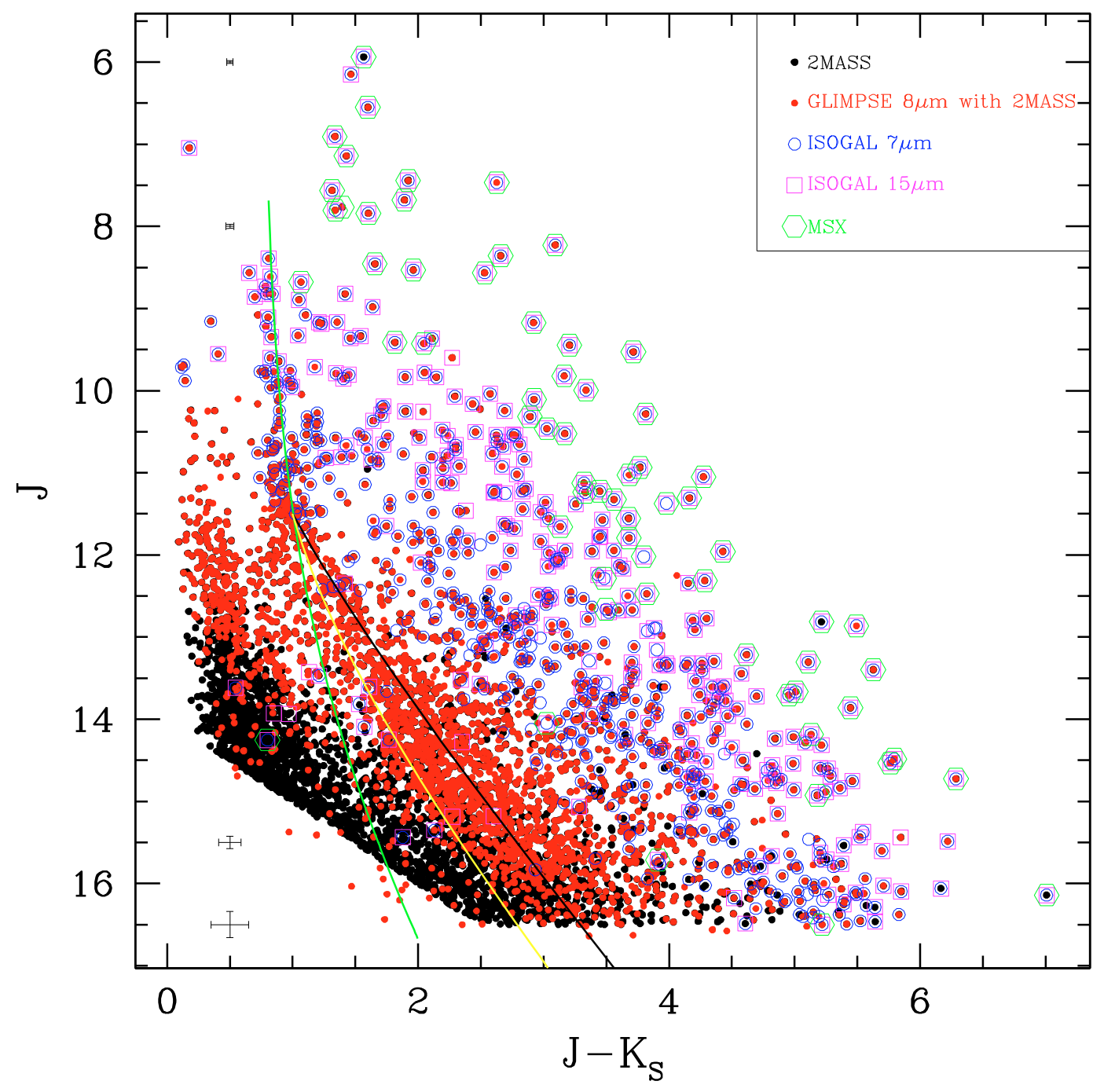

Fig. 4. The $J-K_{\mathrm{S}}$ vs. $J$ CMD. Overlaid are symbols for detections in other surveys as shown in the legend (top right corner). The red-clump locus (RCL) with normal extinction is shown as a solid green curve. The yellow and black curves mark RCL with larger extinction per unit kpc (see Sect. 4). Typical error bars are marked at $J=16.5,15.5,8$ and 6.0 at $J-K_{\mathrm{S}}=0.5$. Online material includes separate figures with sources selected based on detections in the various surveys.

\subsubsection{Associations with $M S X$}

The MSX catalogue in the LN45 field area contains 76 sources, of which only 13 are detected in band $E(21.34 \mu \mathrm{m}) ; 36$ are detected in band $D(14.65 \mu \mathrm{m}) ; 37$ in band $C(12.13 \mu \mathrm{m})$ and 76 in band $A(8.28 \mu \mathrm{m})$. With positional uncertainties of the order of 1" (Egan et al. 2003) 70 of the MSX sources (92\%) are associated with ISOGAL sources within $5^{\prime \prime}$. Two sources with dubious MSX quality flags are associated with rather faint ISOGAL sources.

\subsubsection{Multi-band point source catalogue}

Finally we have a catalogue of 673 reliable $($ flag $=1$ to flag $=$ 5) ISOGAL sources with measurements in the GLIMPSE survey. We also include the 8 additional sources discussed above (with flags 7-9) to get a total of 681 sources. MSX, 2MASS, and DENIS photometry is provided where available. A brief extract of the table is in the online Sect. B. The entire table is available electronically at the CDS. The format of the catalogue with the details of the columns is listed below:

- identification number;
- right ascension;

- declination (from GLIMPSE-2MASS) else ISOGALDENIS if no GLIMPSE-2MASS;

- ISOGAL-DENIS PSC1 name;

- DENIS $I, J, K_{S}$

- 2MASS $J, H, K_{S}$;

- IRAC 1 to IRAC4;

$-[7]$

$-[15]$

- MSX band $A$ to $E$;

- ISOGAL-GLIMPSE association flag;

- type of source (AGB, RGB, YSO, PNe);

- distance (kpc) and $A_{\mathrm{V}}$ (mag) as derived assuming the Red Clump Locus method (see Sect. 4.2) with typical errors;

- $\mathrm{M} K_{\mathrm{S}}$ and $\dot{M}$ for the AGB/RGB (see Sect. 5.1);

- general remarks about the source including comments on validity or otherwise for derived distance.

In Fig. 4, we show the 2MASS CMD $J-K_{\mathrm{S}}$ vs. $J$ with the associated mid infrared sources from different surveys (see also the online Sect. C). Note the varying levels of completeness in the various surveys. The completeness is a function of the 

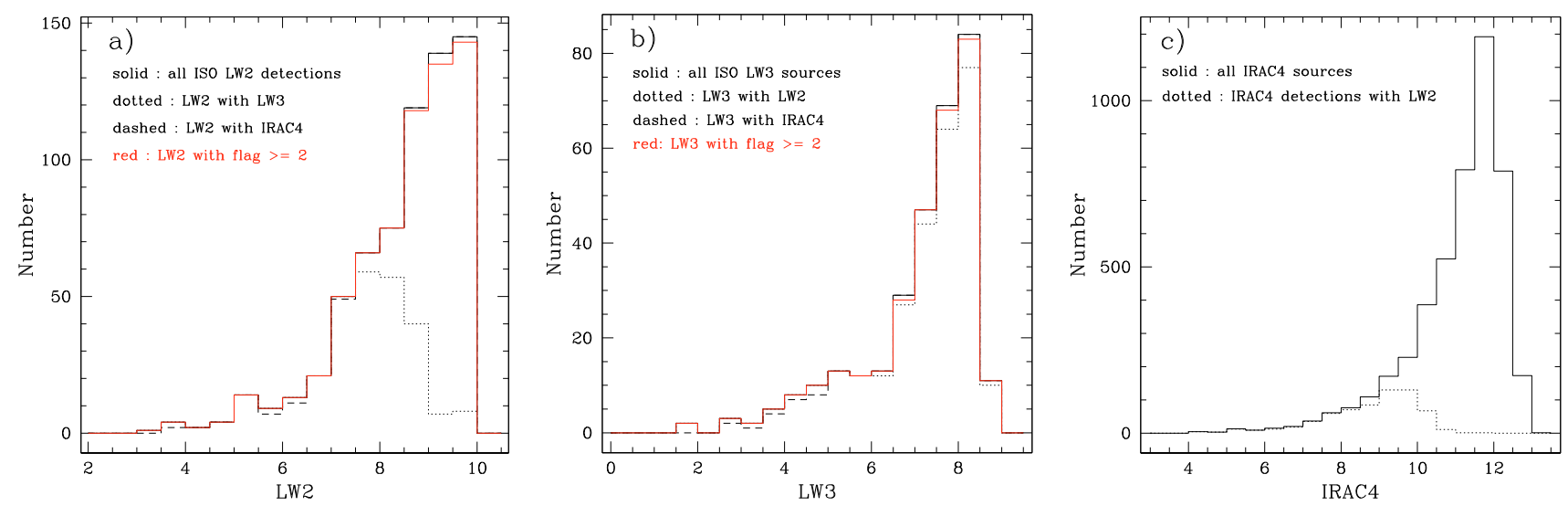

Fig. 5. a) The histogram of the $7 \mu \mathrm{m}$ sources binned by magnitude (binsize $=0.5 \mathrm{mag}$ ) detected in this field with detections also at $15 \mu \mathrm{m}$ (dotted line); detections in GLIMPSE $8.0 \mu \mathrm{m}$ (dashed). In red are shown the $7 \mu \mathrm{m}$ sources with a flag $\geq 2$. b) The histogram of the $15 \mu \mathrm{m}$ sources detected in this field with detections also in $7 \mu \mathrm{m}$ (dotted), and in $8.0 \mu \mathrm{m}$ in GLIMPSE (dashed). $15 \mu \mathrm{m}$ detections with flag $\geq 2$ are shown in red. c) The histogram of the GLIMPSE $8.0 \mu \mathrm{m}$ sources detected in this field with detections also in LW2 $(7 \mu \mathrm{m})$ (dotted).
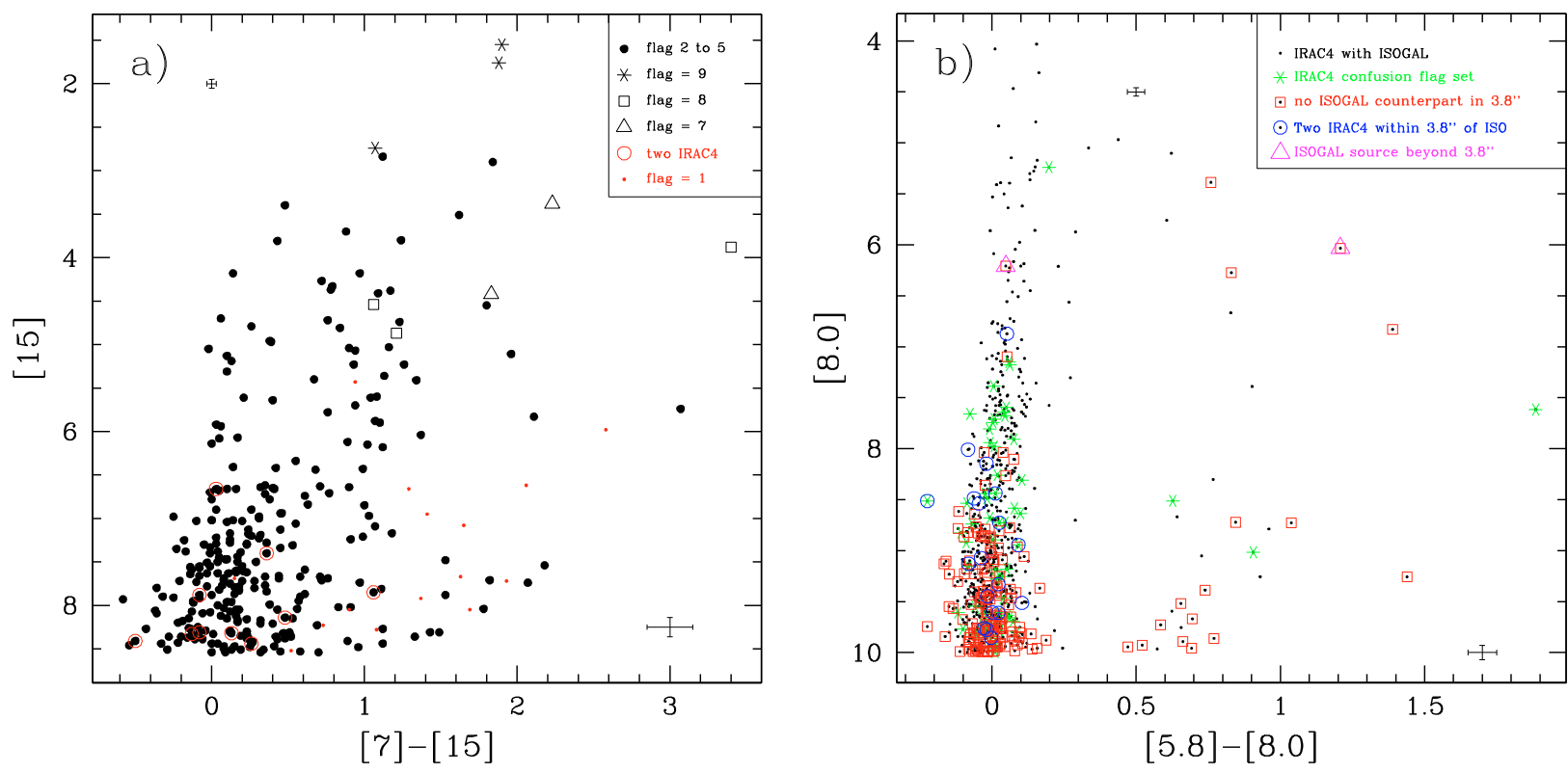

Fig. 6. a) ISOGAL CMD. The sources missed by GLIMPSE due to saturation are marked by an asterisk. Open triangles mark the ISOGAL sources matched to GLIMPSE sources at a greater distance $\left(4.2^{\prime \prime}\right)$ i.e. flag $=7$. Blended/extended ISOGAL sources not in GLIMPSE are shown as open squares. Sources with a second GLIMPSE source within 3.8" are shown with large open circles. b) GLIMPSE CMD showing the sources that are missed by ISOGAL (open squares). We have limited the display to bright GLIMPSE sources (IRAC4 brighter than 10).

wavelength, sensitivity, and the spatial resolution of the surveys. This diagram is discussed in detail in Sect. 4 for source distance and extinction determination.

\section{Accuracy of ISOGAL data}

In this section, we discuss the accuracy of the ISOGAL data using repeated observations (at $15 \mu \mathrm{m}$ ) and using the completeness of identification with GLIMPSE.

\subsection{Comparison with GLIMPSE data}

In Fig. 5 we exhibit the number counts versus magnitude histograms of (a) the $7 \mu \mathrm{m}$ detections (b) the $15 \mu \mathrm{m}$ detections, and (c) the IRAC4 $8 \mu \mathrm{m}$ detections. They show that the ISOGAL detections are $94 \%$ complete up to [7] $=8.5^{m}$ (GLIMPSE $8.0 \mu \mathrm{m}$ magnitude) for the LW2 detections when compared to the GLIMPSE $8 \mu \mathrm{m}$. The $15 \mu \mathrm{m}$ data are complete up to [15] = $7^{m}$ when compared to ISOGAL $7 \mu \mathrm{m}$.

We find that, apart from the 65 rejected sources $(8.7 \%$ of 746 , see Sect. 2.2.1), most of the ISOGAL sources (98.8\% of 681) are detected in GLIMPSE. Three ISOGAL sources below the GLIMPSE saturation limits do not have $8 \mu \mathrm{m}$ counterparts ( flag of 8 and 7) within $3.8^{\prime \prime}$. However, going to a larger association radius retrieves GLIMPSE counterparts for two of the ISOGAL sources (with a corresponding flag of 7). Inspection of the images show that these 5 sources are slightly extended (blended) in the ISOGAL images, while they are resolved by GLIMPSE into individual sources. These 8 sources are shown with different symbols in the ISOGAL CMD of Fig. 6a.

A GLIMPSE colour-magnitude diagram is shown in Fig. $6 \mathrm{~b}$ where we overplot the ISOGAL non-detections with different symbols. As seen, only a small fraction of the bright sources are missed by ISOGAL due to poorer resolution (blends). 

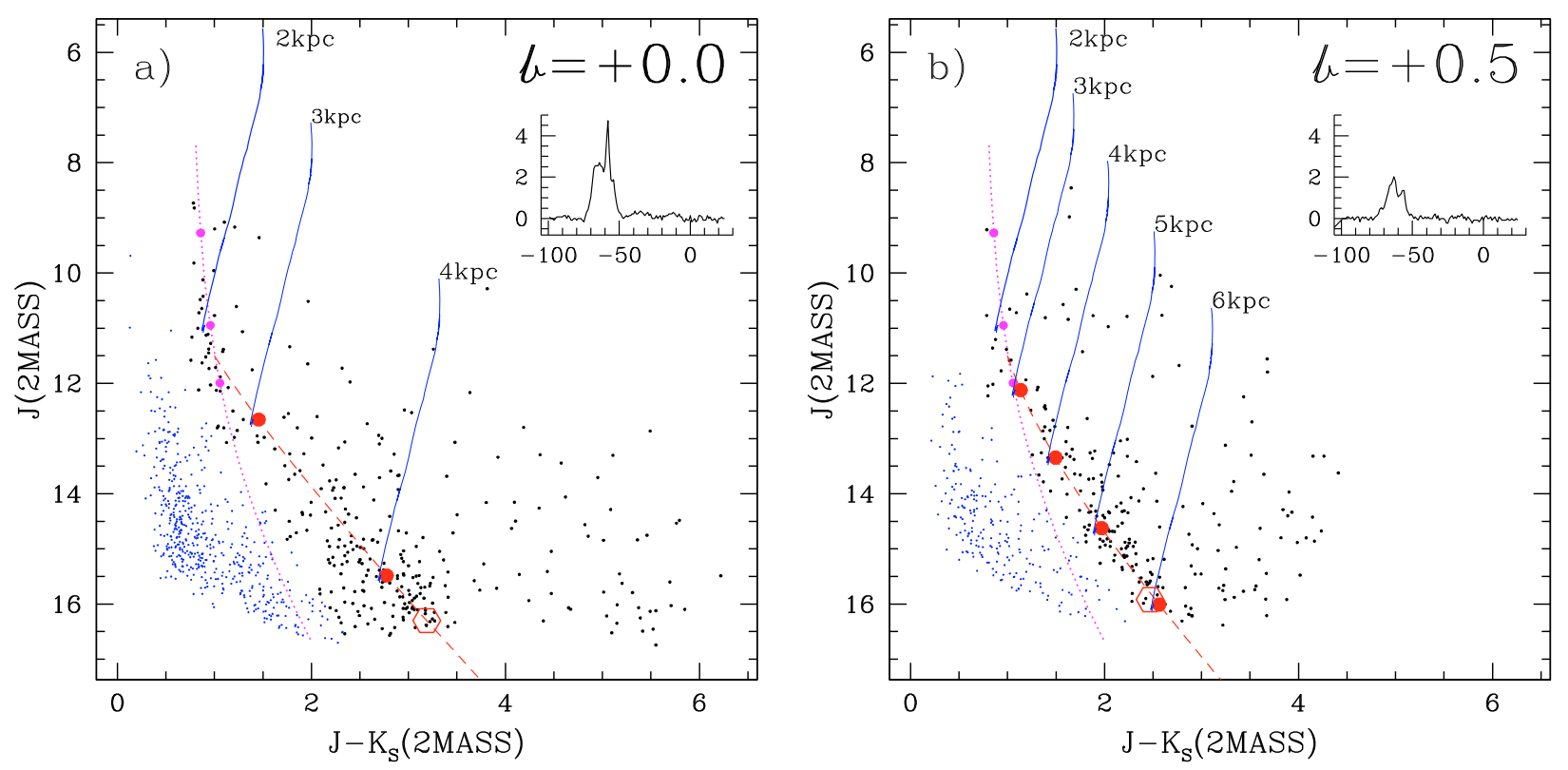

Fig. 7. a) $J$ vs. $J-K_{\mathrm{S}}$ CMD. Blue dots are mostly foreground sources. Black dots are red clump giants and other mid-infrared stellar populations. The red clump locus with normal or uniform extinction $(c J=0.166)$ marked as dotted (magenta) line; extinction as a function of distance marked as a dashed (red) line. This is for the range $-0.0625<b<0.0625$. i.e. $b=0.0$ of Table 3 . Also shown are the RGB/AGB isochrones at distances of $2 \mathrm{kpc}$ (with normal extinction law), and at 3 and $4 \mathrm{kpc}$ (modified law) as labelled in the figure. We use $A_{\mathrm{V}}=6.0\left(A_{J}-A_{\mathrm{K}_{\mathrm{S}}}\right)(\mathrm{Glass} 1999$, and Table 4). b) CMD for the range $0.4375<b<0.5$ i.e. $b=0.5$ of Table 3 . All other details are as mentioned in a). Additional isochrones at 5 and $6 \mathrm{kpc}$ are also shown. In both panels, we show the points listed in Table 3 with large open hexagons. The corresponding CO spectra are also shown as an inset with the velocity scale (in $\mathrm{km} \mathrm{s}^{-1}$ ) marked on the $x$ axis. See the electronic (online) version of the paper for plots corresponding to other values of $b$.

Schuller (2002) finds that extended sources in the ISOGAL inner-bulge fields have relatively larger photometric errors (derived from the fitting of the point-spread function). We note that the blended/extended sources in the LN45 field do not show any similar trends.

\subsection{Repeated $15 \mu \mathrm{m}$ ISOGAL observations}

Repeated ISOGAL $15 \mu$ m observations show that the dispersion (rms) in the $15 \mu \mathrm{m}$ photometry is better than $0.3^{m}$ for the bulk of the sources, while it reaches 0.5 at the fainter end. Three sources are found to be variable in the two epochs of the ISOGAL observations and are discussed in Sect. 5.1.

\section{Interstellar extinction and distance scale}

\subsection{Local value of optical extinction from main-sequence stars}

Field LN45 is located across the Galactic mid-plane where the interstellar extinction is expected to be high. The measurement of interstellar extinction in the optical range is only possible for the nearby regions. Neckel et al. (1980) reported interstellar extinction $A_{\mathrm{V}}$ based on the optical observations of O-type to F-type stars in the direction of $\ell=314^{\circ}$ and $b=0.0^{\circ}$, which is close to LN45 field, to be nearly uniform between 2 and 3 mag up to a distance of $\sim 3.5 \mathrm{kpc}$. This ISOGAL field direction, as already mentioned, crosses the Sagittarius-Carina arm at 1-2 kpc and $15 \mathrm{kpc}$, and it is almost tangential to the Scutum-Crux arm from $\sim 4$ to $10 \mathrm{kpc}$ (Fig. 1). The stars considered in the study by Neckel et al. (1980) belong either to the Sagittarius-Carina arm or the nearby region in front of this arm or just beyond. Determination of the extinction to farther distances must rely on the near-infrared, which is much less absorbed, and the reddening of red giants.

\subsection{Near-infrared CMDs of red giants, extinction, and distance scales}

Figure 7 shows the most useful diagram for the purpose of the determination of the distance and extinction in the direction of LN45 - the CMDs $J-K_{\mathrm{S}}$ vs. $J$ towards two different ranges in galactic latitude as mentioned in the captions. The red clump sequence is clearly seen in Figs. $7 \mathrm{a}$ and $7 \mathrm{~b}$. The red clump sequence can be identified at $J$ of $11^{m}$ and $J-K_{\mathrm{S}}$ of 1 . Going towards fainter $J$, their $J-K_{\mathrm{S}}$ also becomes redder and at the base of the CMD reaches $J-K_{S}$ beyond $2.5^{m}$. As discussed e.g. by Indebetouw et al. (2005) for a similar line of sight (see also López-Corredoira et al. 2002), the red clump giants can be isolated and their distribution fitted to determine the extinction with distance. In the diagrams of Fig. 7, the red clump giants are isolated from the foreground main sequence stars, mostly numerous, faint, cool dwarfs, and the background more luminous giants up to the RGB tip and the upper AGB. The latter are discussed below in Sect. 5. Note that the analysis in this section is based on the entire 2MASS catalogue for this field.

In a large field such as LN45, the extinction may vary significantly because of the range of galactic latitudes and the clumpiness of molecular clouds. With the very large number of clump stars available, one can try to use them to trace the extinction in a number of smaller, more homogeneous spatial cells tiling the whole field.

To define an approximate distance scale, we first assume that the average extinction in each field is just proportional to the distance $d$, and fit a mean curve for the red clump locus (RCL) 
Table 3. $A_{\mathrm{V}}(\mathrm{mag})$ and distance (pc) derived from red clump at the bottom end of the $J$ vs. $J-K_{\mathrm{S}} \mathrm{CMD}$. Also shown are corresponding values of $J$, $J-K_{\mathrm{S}}$, and the $A_{\mathrm{V}}$ derived from the CO spectral survey of Dame et al. (1987) and the corresponding $A_{\mathrm{V}}$ and distance from the Besançon Model (BM) (Robin et al. 2003). All the cells are taken centred at $\ell=315.0$ between the two $b$ values $\left(b_{\text {low }}, b_{\text {high }}\right)$ shown.

\begin{tabular}{|c|c|c|c|c|c|c|c|c|c|c|c|}
\hline$b$ & $b_{\text {low }}$ & $b_{\text {high }}$ & $J$ & $\overline{J-K_{\mathrm{S}}}$ & $A_{\mathrm{V}}$ & Distance & $A_{\mathrm{V}}(\mathrm{CO})$ & $J(\mathrm{BM})$ & $J-K_{\mathrm{S}}(\mathrm{BM})$ & $A_{\mathrm{V}}(\mathrm{BM})$ & distance(BM) \\
\hline 0.5 & 0.4375 & 0.5 & 16.02 & 2.57 & 11.78 & 6008 & 7.36 & 16.08 & 3.03 & 12.18 & 5310 \\
\hline 0.375 & 0.3125 & 0.4375 & 15.92 & 2.70 & 12.66 & 5184 & 8.80 & 15.93 & 2.86 & 11.30 & 5050 \\
\hline 0.25 & 0.1875 & 0.3125 & 16.07 & 2.84 & 13.54 & 4990 & 11.51 & 16.04 & 3.07 & 12.80 & 5490 \\
\hline 0.125 & 0.0625 & 0.1875 & 16.11 & 3.03 & 14.78 & 4410 & 13.81 & 16.05 & 3.00 & 12.53 & 5090 \\
\hline 0.0 & -0.0625 & 0.0625 & 16.30 & 3.19 & 15.83 & 4250 & 15.14 & 16.21 & 3.40 & 14.34 & 5510 \\
\hline-0.125 & -0.1875 & -0.0625 & 16.23 & 3.12 & 15.39 & 4331 & 16.02 & 16.22 & 3.30 & 13.90 & 5410 \\
\hline-0.25 & -0.3125 & -0.1875 & 16.19 & 2.98 & 14.42 & 4750 & 11.88 & 16.25 & 3.22 & 13.66 & 5050 \\
\hline-0.5 & -0.5 & -0.4375 & 15.92 & 2.47 & 11.13 & 6194 & 9.64 & 15.92 & 2.55 & 9.80 & 5910 \\
\hline
\end{tabular}

following the method of Indebetouw et al. (2005) and their Eqs. (2) and (3):

$$
\begin{aligned}
& J=M_{J}+5 \log (d / 10 \mathrm{pc})+c_{J}(d / 10 \mathrm{pc}) \\
& J-K_{\mathrm{S}}=M_{J}-M_{K \mathrm{~s}}+\left(c_{J}-c_{K \mathrm{~s}}\right)(d / 10 \mathrm{pc})
\end{aligned}
$$

where $c_{J}$ and $c_{K \mathrm{~s}}$ are the average extinction per unit distance in the $J$ and $K_{\mathrm{S}}$ bands. We assume the same standard values as used by Indebetouw et al. (2005) and López-Corredoira et al. (2002) (see also Wainscoat et al. 1992) for the mean absolute magnitudes of clump stars: $M_{J}=-0.95, M_{K \mathrm{~s}}=-1.65, J_{0}-K_{\mathrm{S} 0}=0.75$, and extinction ratios: $c_{J} / c_{K s}=A_{J} / A_{K \mathrm{~s}}=2.5$. The curve of the red clump locus then depends only on the extinction parameter $c_{J}$ to be fitted.

However, it is clear from Fig. 7 that such a simple curve with a single value of $c_{J}$ for each subfield cannot properly fit the actual red clump locus. This is not surprising since the variation of extinction with distance, represented by $c_{J}$, is much smaller (by factors $\sim 2$ to 7 ) locally than within the Scutum-Crux spiral arm, especially where the molecular clouds are located.

For defining such cells, it seems logical to use the galactic latitude, $b$, limits corresponding to the beams of the $\mathrm{CO}$ survey of Dame et al. (1987), in order to find a correlation with the specific extinction of the molecular clouds inferred from the CO intensity. Nine CO spectra nearly cover the LN45 $b$ range completely and we have defined nine cells corresponding to the $b$ range of each beam. These are tabulated in Table 3. The $\mathrm{CO}$ spectra are inset in Fig. 7.

For each cell, we got a reasonable fit of the global RCL with the following procedure. We first determined the best value of $c_{J}$ for the two ends of the curve: the local one, and the one for the largest extinctions available in each group corresponding to some location in the Scutum-Crux arm. The combination of these two segments provide a reasonable fit for more than half of the curve in all cases. By inspection we determined the value of $J-K_{\mathrm{S}}$ (or of $d$ or $A_{\mathrm{V}}$ ) for which the considered segment is an acceptable approximation, and we finished by joining the two segment ends by a linear interpolation in $c_{J}$. The distance and $A_{\mathrm{V}}$ at the farther end of the RCL and the corresponding $J$, $J-K_{\mathrm{S}}$ are listed in Table 3 along with the $A_{\mathrm{V}}$ derived from the $\mathrm{CO}$ strength. Values obtained from the Besançon model of the Galaxy (Robin et al. 2003) are also included in this table for comparison. All the values of $c_{J}$ for the local segments are the same $\left(c_{J}=0.166\right)$. The uncertainties in $d, c_{J}$ and $c_{K \mathrm{~s}}$ are about $500 \mathrm{pc}, 0.1 \mathrm{mag} / \mathrm{kpc}$, and $0.03 \mathrm{mag} / \mathrm{kpc}$, respectively.

Table 3 and Fig. 8 compare the extinction and distances derived with those from the Besançon model. This model implements the Marshall et al. (2006) 3D extinction map. We note that the spatial resolution of the Marshall et al. (2006) map is $15^{\prime} \times 15^{\prime}$. Figure 8 shows small discrepancies between our derived extinction values and distances compared to the values from the model. However, considering the fact that the model relies on many parameters such as scale length and height of the thin and thick discs, metallicity distribution, etc., the discrepancies are not significant.

For the highest latitudes, $|b| \sim 0.5^{\circ}$, it is seen that the distances traced by the RCL range up to $7 \mathrm{kpc}$ (see Fig. 8a), i.e. over a substantial part of the Scutum-Crux arm. However, at the lowest latitudes, $|b|<0.2^{\circ}$, only red clump stars in the nearest part of the Scutum-Crux arm, at $4-5 \mathrm{kpc}$, are detected in $J$ by 2MASS. The largest $A_{\mathrm{V}}$ seen in this field is close to $30 \mathrm{mag}$ for stars close to the RGB tip with $J-K_{\mathrm{S}} \sim 6 \mathrm{mag}$.

In the various CMDs, e.g. $J$ vs. $J-K_{\mathrm{S}}$, for each distance (and reddening) determined from the red clump, one may trace the corresponding isochrone of the red giants of various luminosity (see e.g. Fig. 7), assuming it is uniquely defined and, in particular, that these stars have approximately the same age and metallicity in a given direction. For each ISOGAL red giant, including most AGB stars without too strong mass loss, one may thus determine its distance and luminosity by tracing the corresponding isochrone (Bertelli et al. 1994) from the position of the source in the plot and considering its intersection with the red clump locus (Fig. 7). This method applies to the majority of ISOGAL sources since they all are such red giants. In the absence of other information about the nature of the source, we have used it systematically for all ISOGAL sources, at least as a first approximate step. However, it should obviously be modified and adapted to the actual nature of the sources, in particular for the three other main classes apart from the red giants:

1) Main sequence stars; ISOGAL detects them only at very short distances (e.g. $\sim 100 \mathrm{pc}$ for a K0V star, $\sim 250 \mathrm{pc}$ for an F3V star, $\sim 300$ pc for a B9 star). They thus have low reddening, and bluer intrinsic colours or lower luminosity than red clump stars. The most obvious cases among ISOGAL sources are a few sources located below the red clump locus or to the left of the $A_{\mathrm{V}}=0$ isochrone of red giants in Fig. 7.

2) Young stars; class II and III protostars have intrinsic nearIR colours not very different from that of red giants, so that de-reddening them as if they were red giants, may give sufficient constraints on their distance and luminosity. However, being embedded in dust adds to the uncertainty in estimating the extinction and distance to many of these sources.

3) AGB stars with enough mass loss to modify $\left(J-K_{\mathrm{S}}\right)_{0}$ with respect to the isochrone by significant circumstellar dust absorption $\left(\dot{M}>\sim 10^{-6} M_{\odot} / \mathrm{yr}\right)$. A few such sources may be identified easily from $K_{\mathrm{S}}-[15]$ (see Sect. 5.1 and Table B.1), 

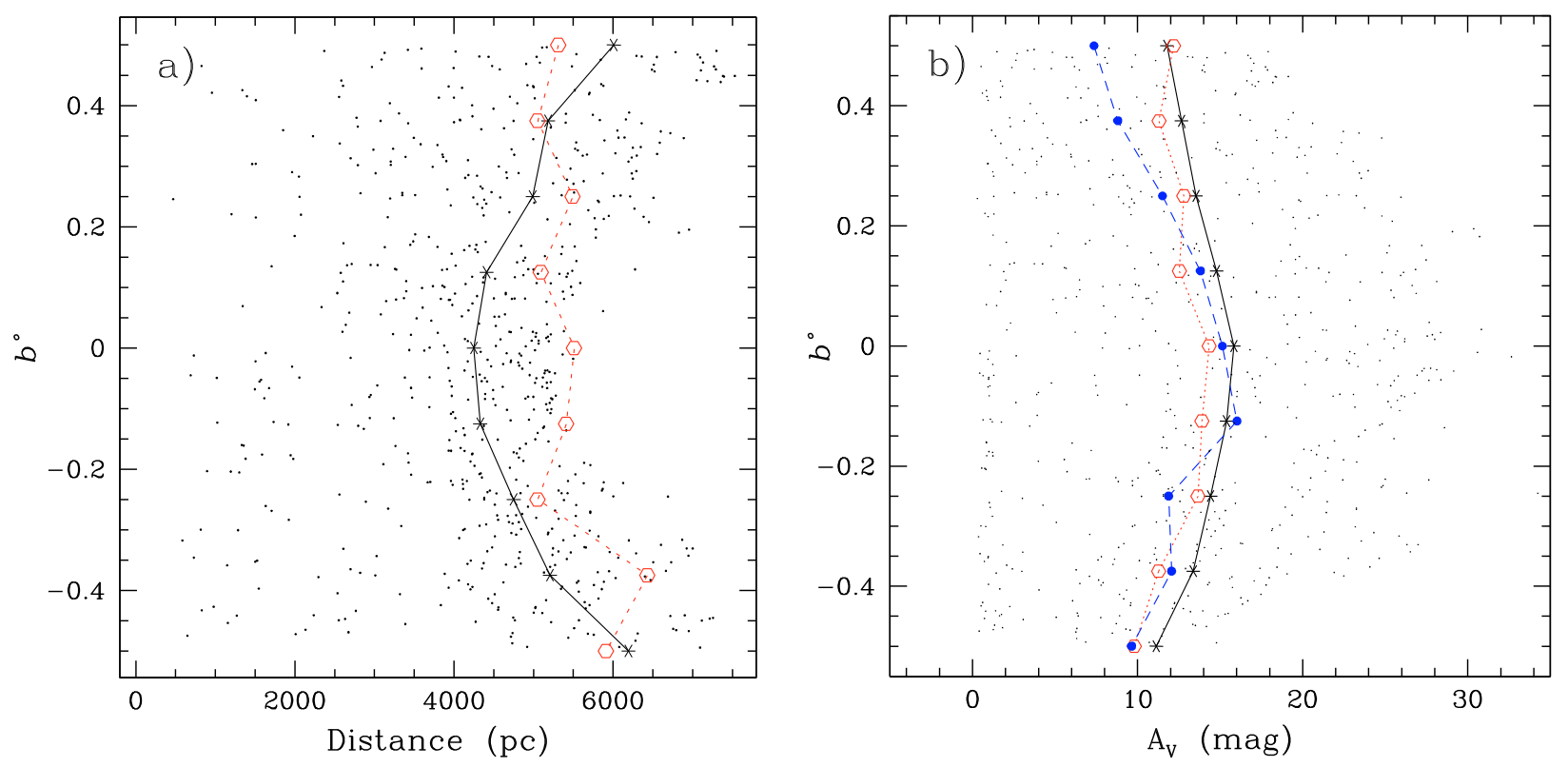

Fig. 8. a) Plot showing the derived positions for the sources as a function of galactic latitude $b$. The solid line (black) connecting the asterisks represents the location of the red clump at the base of the CMD. The dotted line (red) represents the corresponding red clump location from the Besançon model (Robin et al. 2003). b) Derived $A_{\mathrm{V}}$ as a function of galactic latitude. The solid line (black) represents the $A_{\mathrm{V}}$ at the position of the red clump in the observed CMDs. The dotted line (red) represents the values from the Besançon model while the dashed line (blue) is the $A_{\mathrm{V}}$ derived from the ${ }^{12} \mathrm{CO}$ as listed in Table 3.

but disentangling their interstellar and circumstellar extinction remains difficult, which adds uncertainty to their distance and luminosity determination.

A special case occurs for the most distant ISOGAL stars (mostly AGB) detected in $J$ and $K_{\mathrm{S}}$ but for which the isochrone would cut the red clump locus beyond the range where it is detected (Fig. 7). However, one may still infer an approximate value of their distance by noticing that the isochrones actually cutting the $\mathrm{RCL}$, have approximately constant $\delta\left(J-K_{\mathrm{S}}\right) / \delta d$ spacing. One may thus extrapolate this to draw isochrones at larger distances (Fig. 7).

Keeping these difficulties in mind, we give an estimate in Table B. 1 of the reddening and distance to the ISOGAL sources with detections in $J$ and $K_{\mathrm{S}}$, assuming that they are red giants fitting the standard isochrone of Bertelli et al. (1994), except for those having very large mass loss as discussed in Sect. 5.1, and those identified as young stars (see Sect. 5.2), as well as the foreground main sequence. It is easy to infer the luminosity $L_{\mathrm{bol}}$ from $K_{\mathrm{S} 0}$ with the well-established bolometric correction, $\mathrm{BC}_{K}$, for such red giant stars close to 3 (see e.g. Frogel \& Whitford 1987) and Fig. 6 of Ojha et al. (2003).

\subsection{Variations in extinction across the field and correlation with $\mathrm{CO}$}

As discussed above (Sect. 4.2), the extinction is not uniformly proportional to the distance in the line of sight. The total extinction may be attributed to two main components - the first proportional to the average interarm column density of dust. The second component of extinction is localised and associated with the excess of interstellar molecular gas in the spiral arms.

The effect of extinction in the Scutum-Crux arm should be responsible for the irregular patterns seen in the distribution of red clump stars along the red clump locus in the individual plots of each cell in Fig. 7 (see also the online material). The absence of stars in some sections of these loci should correspond to localised molecular clouds, with a jump in $J-K_{\mathrm{S}}$ and thus in $A_{\mathrm{V}}$ on a very short distance where there are very few red clump stars; on the other hand, the sections with a regular large density of stars should correspond to regular smooth increase in $A_{\mathrm{V}}$.

\subsection{Extinction law at 7 and $15 \mu \mathrm{m}$ and in the GLIMPSE bands}

Jiang et al. (2003, 2006) have estimated the interstellar infrared extinction for the ISOGAL fields based on the assumption that most of the sources detected by ISOGAL are luminous RGB or AGB stars with moderate mass loss. Another assumption is that the intrinsic colours $\left(J-K_{\mathrm{S}}\right)_{0}$ and $(K-[7])_{0}$ are similar for RGB and AGB stars, except for the sources with high mass loss. Jiang et al. (2003) have discussed this point in detail. In $\left(J-K_{\mathrm{S}}\right)$ vs. $\left(K_{\mathrm{S}}-[7]\right)$ colour-colour diagrams (CCD) most of the sources then follow a straight line with some dispersion. The fitting of this straight line thus determines the ratio $\left[A_{K \mathrm{~s}}-A_{[7]}\right] /\left[A_{J}-A_{K \mathrm{~s}}\right]$. We repeated the fitting of Jiang et al. (2006) independently for the LN45 field (Fig. 9a) and found $\left(A_{K \mathrm{~s}}-A_{[7]}\right) /\left(A_{J}-A_{K \mathrm{~s}}\right)=0.35 \pm 0.02$, in agreement with their value. Similarly, we have found $\left(A_{K \mathrm{~s}}-A_{[15]}\right) /\left(A_{J}-A_{K \mathrm{~s}}\right) \sim 0.39$ (see Fig. 9b), with a larger uncertainty because of the increased effect of mass loss on $\left(K_{\mathrm{S}}-[15]\right)_{0}$ (see below and Jiang et al. $2003,2006)$. Figure 9c displays the $\left(J-K_{\mathrm{S}}\right)$ vs. $\left(K_{\mathrm{S}}-[8.0]\right)$ figure for the GLIMPSE IRAC4 band. A similar study has been done by Indebetouw et al. (2005), and they present extinction in the GLIMPSE IRAC bands ( $A_{\text {IRAC }}$ ) using similar diagrams towards a different GLIMPSE field in the Galactic disc. The values derived for $\left(A_{K \mathrm{~s}}-A_{\lambda}\right) /\left(A_{J}-A_{K \mathrm{~s}}\right)$ for the LN45 field are tabulated in Table 4 and are consistent with Jiang et al. (2006) and Indebetouw et al. (2005). Note, however, that different extinction coefficients have been derived by various authors depending on the line of sight. For example, Flaherty et al. (2007) derived different extinction coefficients over a wide range of near and mid-infrared wavelengths towards nearby star-forming regions. 

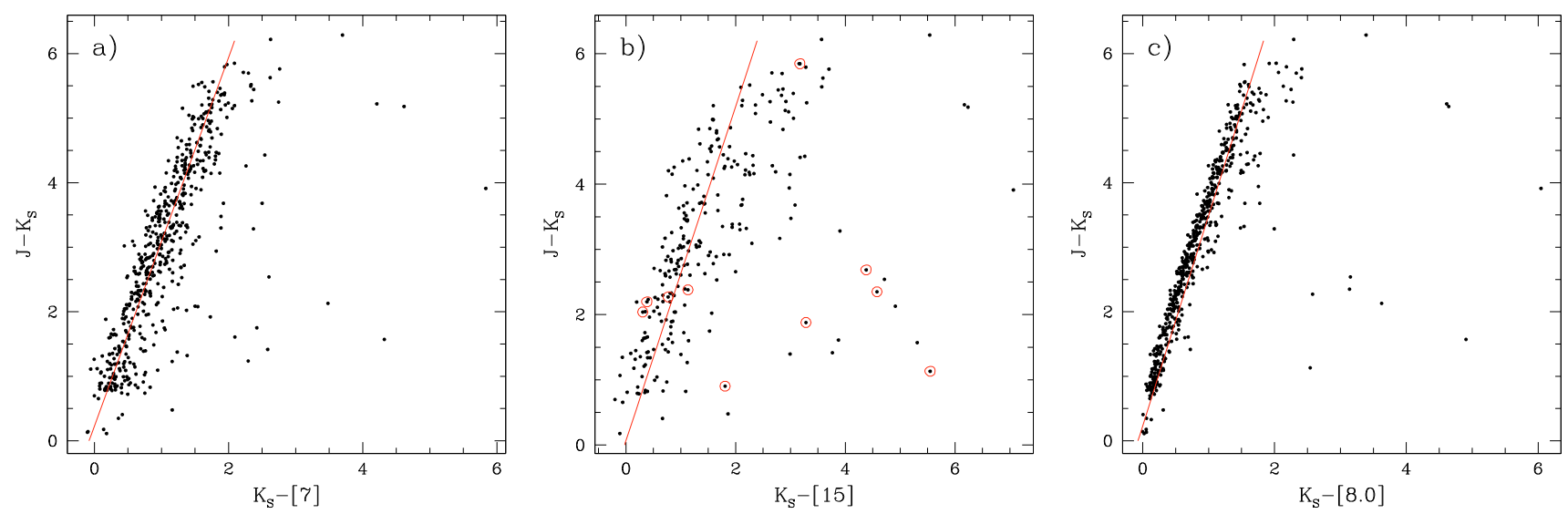

Fig. 9. a) $J-K_{\mathrm{S}}$ vs. $K_{\mathrm{S}}-[7] \mathrm{CCD}$ showing the fit used to determine $\left(A_{K_{\mathrm{s}}}-A_{[7]}\right) /\left(A_{J}-A_{K \mathrm{~s}}\right)$, b) $J-K_{\mathrm{S}}$ vs. $K_{\mathrm{S}}-[15]$ CCD showing the fit used to determine $\left(A_{K \mathrm{~s}}-A_{[15]}\right) /\left(A_{J}-A_{K \mathrm{~S}}\right)$. Sources not seen at [7] are shown with additional open circles. c) $J-K_{\mathrm{S}}$ vs. $K_{\mathrm{S}}-$ [8.0] CCD showing the fit used to determine $\left(A_{K \mathrm{~s}}-A_{[8.0]}\right) /\left(A_{J}-A_{K \mathrm{~s}}\right]$. Only sources with ISO counterparts are shown here.

Table 4. $A_{\lambda} / A_{\mathrm{V}}$ for different filters.

\begin{tabular}{ccccl}
\hline \hline Filters & $\lambda_{\mathrm{c}}$ & $\Delta \lambda$ & $A_{\lambda} / A_{\mathrm{V}}$ & Notes \\
\hline$J$ & 1.25 & 0.25 & 0.256 & 2MASS \\
$H$ & 1.65 & 0.3 & 0.142 & 2MASS \\
$K_{\mathrm{S}}$ & 2.17 & 0.32 & 0.089 & 2MASS \\
$L W 2$ & 6.7 & 3.5 & 0.031 & ISOGAL \\
$L W 3$ & 14.3 & 6 & 0.024 & ISOGAL \\
$I R A C 1$ & 3.58 & 0.75 & 0.046 & GLIMPSE \\
$I R A C 2$ & 4.50 & 1.02 & 0.042 & GLIMPSE \\
$I R A C 3$ & 5.80 & 1.43 & 0.036 & GLIMPSE \\
$I R A C 4$ & 8.00 & 2.91 & 0.037 & GLIMPSE \\
\hline
\end{tabular}

They also obtained slightly different numbers for the data analysed by Indebetouw et al. (2005). Flaherty et al. (2007) obtained $A_{[24]} / A_{\mathrm{V}}$ values of 0.039 to 0.046 towards two star-forming regions and these are higher than the value we have obtained for $A_{[15]} / A_{\mathrm{V}}(=0.024)$. Román-Zúñiga et al. (2007) find larger extinction coefficients for the IRAC filters towards a dark cloud core.

\section{Nature of sources}

Figure 10a shows the $\left(K_{\mathrm{S}}-[15]\right)_{0}$ vs. $M_{K \mathrm{~s} 0}$ diagram for stars to which the distance has been derived. This implies that these sources have $J$ and $K_{\mathrm{S}}$ counterparts. Theoretical tip of the $\mathrm{RGB}$, as well as the position of the red clump provided by the isochrones, are also indicated. The bulk of the sources are RGB stars, while a few K giants are also present in our sample.

One interesting source (number 729 in the LN45 catalogue) has an extremely large $\left(K_{\mathrm{S}}-[15]\right)_{0}>8$. It is very bright at [7] and at [15]. In the [7] vs. $K_{\mathrm{S}}-$ [7] diagram (Fig. 13c), this object is situated at a location $\left([7]=3.64, K_{\mathrm{S}}-[7]=7.38\right)$ populated by high mass-losing AGB stars (Schultheis et al. 2000). The derived distance of $5 \mathrm{kpc}$ for this object is certainly too low (due to uncertain 2MASS $J$ band photometry), which means that the absolute magnitude must be much brighter. This source is not shown in the Fig. 10a due to its distance being very uncertain.

ISOGAL [7]-[15] vs. [15] CMD is shown in Fig. 10b, where the various stellar populations detected by ISOGAL are plotted with different symbols. This figure is discussed further in the Sect. 5.2.

ISOGAL-P J143318.1-604938 (number 636 in the LN45 catalogue) is actually the well known planetary nebula
ESO 134-7 (Kerber et al. 2003). This planetary nebula is notable for its exceptionally high velocity wind and [WN] nucleus (Morgan et al. 2003). This has been mis-identified as a YSO candidate by Felli et al. (2002). This source is marked in Fig. 10b and does not appear in Fig. 10a due to uncertain distance.

\subsection{AGB stars and mass loss}

\subsubsection{Identification, distance, variability, dereddening, and luminosity of AGB stars}

Figure 11a shows the $\left(K_{\mathrm{S}}-[15]\right)_{0}$ vs. [15] diagram of the LN45 field. Only those sources believed to be AGB or RGB stars with derived distances are plotted, so that young stellar objects are not included. As discussed by Omont et al. (1999) and Glass et al. (1999), this diagram represents a mass-loss sequence. We superimposed objects that are variable in $K_{\mathrm{S}}$ and $J$ (based on the 2MASS and the DENIS data that were observed at different epochs) with an amplitude larger than 0.4 mag in $J$ and 0.2 mag in $K_{\mathrm{S}}$. We found in total 5 variable AGB stars that populate the typical region of long-period variables (LPV) (see also Glass et al. 1999) and one object (number 551 in Table B.1) with an absolute magnitude $\mathrm{M} K_{\mathrm{S}}=-5$ below the RGB tip. Its colour in $\left(K_{\mathrm{S}}-[15]\right)_{0}$ suggests a red giant classification, and as it has low (relatively bluer) $J-K_{\mathrm{S}}$ colour, the star is expected to be nearby. A total of 3 sources (numbers 233, 424, and 729 in Table B.1) were found to be variable (amplitude $>0.5 \mathrm{mag}$ ) in the repeated $15 \mu \mathrm{m}$ observations. This variable identification has been inferred from observations of only two epochs. Therefore, the actual number of LPVs is certainly much greater, particularly the sources with luminosities higher than the RGB tip. Schultheis et al. (2000) claim that about $40 \%$ of the variable stars can be recovered using only two epoch measurements.

Figure $11 \mathrm{~b}$ shows the luminosity function in the $\mathrm{M} K_{\mathrm{S}}$ band for the RGB and AGB stars. The tip of the RGB and the red clump are indicated. The peak in the general distribution is at $\mathrm{M} K_{\mathrm{S}}=-5$, while those sources with $15 \mu \mathrm{m}$ ISOGAL counterpart peak at one magnitude brighter. This is due to the reduced sensitivity and completeness of the $15 \mu \mathrm{m}$ flux. We detect all AGB stars at $15 \mu \mathrm{m}$ in this direction, while the $15 \mu \mathrm{m}$ sources become incomplete just below the RGB-tip. 

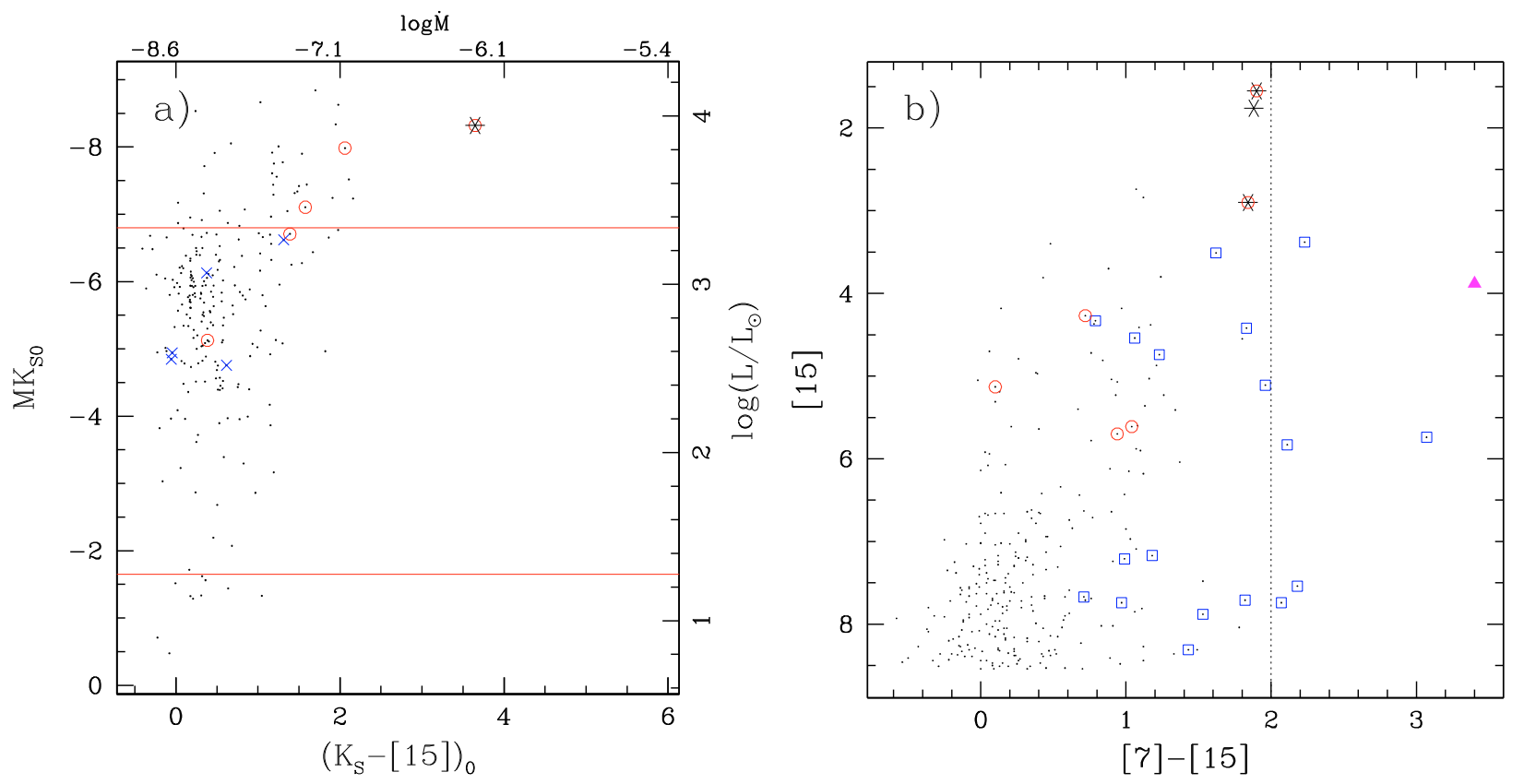

Fig. 10. a) $\mathrm{M} K_{\mathrm{S} 0}$ vs. $\left(K_{\mathrm{S}}-[15]\right)_{0}$ diagram. Horizontal lines at $\mathrm{M} K_{\mathrm{S} 0}$ of -6.8 and -1.65 show the RGB tip and the red clump magnitudes, respectively. Variables in [15] are marked by asterisks. Variables in $K_{\mathrm{S}}$ are represented by open (red) circles. Non-detections at [7] have additional blue crosses. Mass-loss rate $(\log \dot{M})$ is marked for corresponding colours on the top axis. See Sect. 5.1. Luminosities are labelled on the right axis. YSO candidates are not shown b). The ISOGAL [7]-[15] vs. [15] CMD showing the various types of sources: variables (15 $\left.\mu \mathrm{m}, K_{\mathrm{S}}\right)$, YSO, planetary nebula (magenta triangle, see text). YSO candidates are marked with blue open squares. All other symbols are as in a).
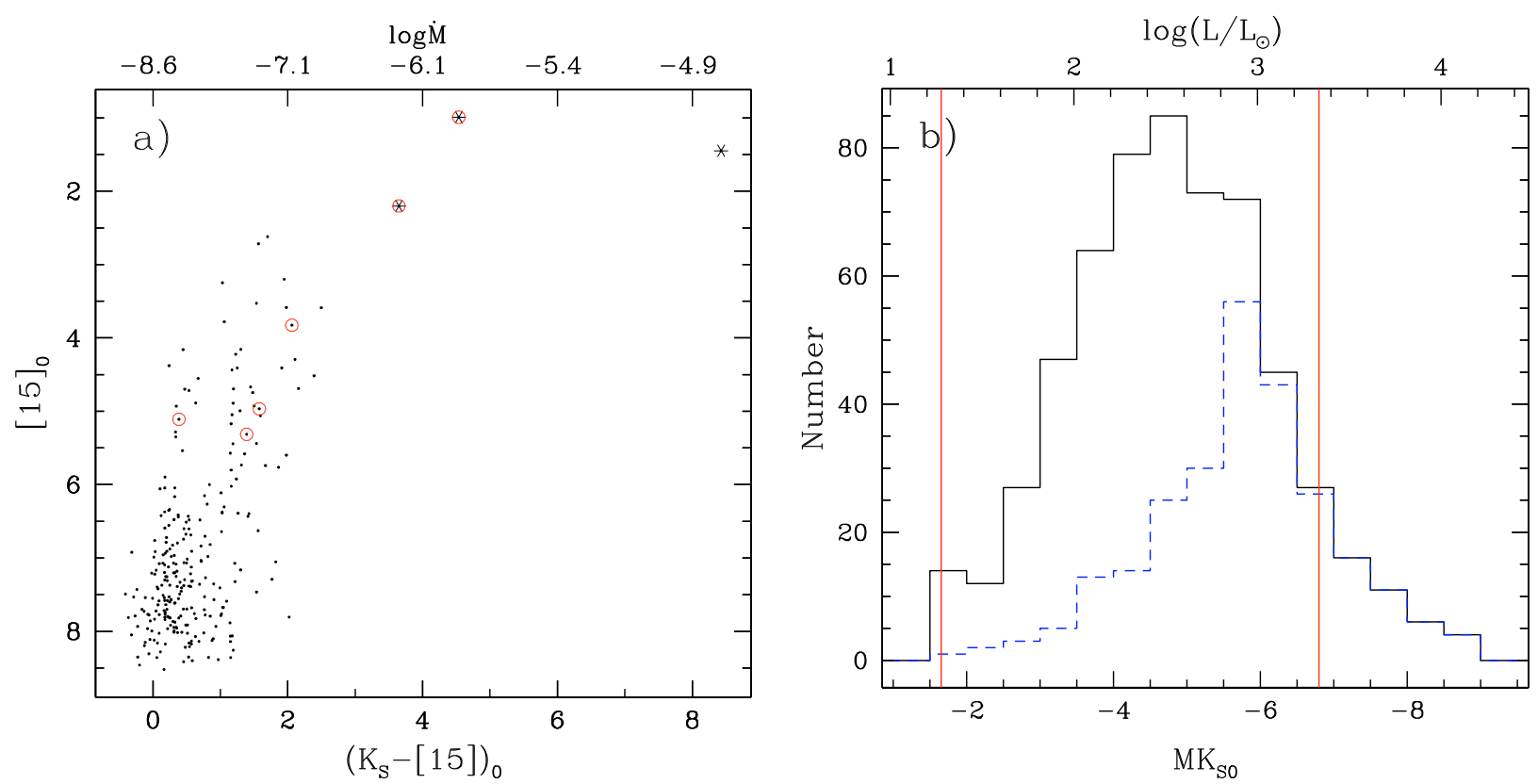

Fig. 11. a) The $\left(K_{\mathrm{S}}-[15]\right)_{0}$ vs. [15] diagram. Candidate variable stars from the near-infrared photometry (see text) are shown as (red) open circles. Asterisks are sources with more than $0.5 \mathrm{mag}$ difference in the two [15] observations. The top axis is labelled with values of log $\dot{M}$. We have included the 2MASS photometry for the sources saturated in GLIMPSE. Note that YSOs and the known planetary nebula are not included in the plot. b) Histogram of $\mathrm{M} K_{\mathrm{S}}$ in bins of $0.5 \mathrm{mag}$ for sources brighter than the red clump $\left(\mathrm{M} K_{\mathrm{S} 0}=-1.65\right)$. Solid line shows the histogram for all ISOGAL sources with 2MASS counterparts (including sources saturated in GLIMPSE and those with $J$ band missing in GLIMPSE-2MASS). Dashed (blue) line is the histogram for the sources that have an ISOGAL $15 \mu \mathrm{m}$ counterpart. YSOs and the known planetary nebula are not included. The red clump and the tip of the RGB are shown as solid vertical lines at $\mathrm{M} K_{\mathrm{S} 0}$ of -1.65 and -6.8 , respectively.

\subsubsection{Mass-loss rate determination from $15 \mu \mathrm{m}$ excess}

It is generally agreed that the mid-infrared excess $\left(\left[\lambda_{1}\right]-\right.$ $\left.\left[\lambda_{2}\right]\right)_{0}$ is a good indicator of the mass-loss rate $\dot{M}$ of AGB stars (Whitelock et al. 1994; Le Bertre \& Winters 1998;
Ojha et al. 2003. Ojha et al. (2003) show that $\left(K_{\mathrm{S}}-[15]\right)_{0}$ provides a good estimate of $\dot{M}$, and based on this they have determined $\dot{M}$ of AGB stars in the ISOGAL fields of the intermediate bulge (see also Alard et al. 2001). Ojha et al. (2003) discussed two models to relate AGB infrared colours to mass-loss rates. 
We used dust radiative transfer models by Groenewegen (2006) for oxygen-rich AGB stars. The mass-loss rates are based on a model for an oxygen-rich AGB star with $T_{\text {eff }}$ of $2500 \mathrm{~K}$ and $100 \%$ silicate composition. From the set of values of $\dot{M}$ computed by Groenewegen (2006), we used the following relation between $\left(K_{\mathrm{S}}-[15]\right)_{0}$ and the mass-loss rate $\dot{M}$

$\log \dot{M}=-8.6171+0.85562 X-0.064143 X^{2}+0.0018083 X^{3}(3)$

where $\dot{M}$ is in $M_{\odot} \mathrm{yr}^{-1}$ and $X$ is $\left(K_{\mathrm{S}}-[15]\right)_{0}$. We derived massloss rates for ISOGAL sources of LN45 field from this relation, and the mass-loss rates are listed in Table B.1. This relation is valid for $-10.0<\log \dot{M}<-4.7$, but the accuracy of our data limits its use to $\dot{M}>10^{-8} M_{\odot} \mathrm{yr}^{-1}$.

Figure 12 shows the histogram of the derived values of the mass-loss rates of the AGB stars in the LN45 field. Most of the sources are RGB stars with $\left(K_{\mathrm{S}}-[15]\right)_{0}<1$ (i.e. $\log \dot{M}<$ -7.8). Jiang et al. (2003) studied a similar field at $\ell=-18 \mathrm{deg}$ $(\mathrm{FC}-01863+00035)$. Their $\left(K_{\mathrm{S}}-[15]\right)_{0}$ vs. [15] diagram looks similar to ours.

In most cases, the value of $\left(K_{\mathrm{S}}-[15]\right)_{0}$ is derived from the observed $K_{\mathrm{S}}$ and $15 \mu \mathrm{m}$ magnitudes and by applying the reddening values previously determined from the red giant isochrone. However, one should note that an appreciable error in the value of $\left(K_{\mathrm{S}}-[15]\right)_{0}$ may result for stars with high mass-loss rates as $K_{\mathrm{S}}$ and $15 \mu \mathrm{m}$ observations are from different epochs and such stars are strong variables.

The overall observational uncertainty is rather large for the derived values of $\dot{M}$ for individual stars. The combined errors in photometry and reddening determination and the effect of variability result in a global $\mathrm{rms}$ uncertainty of $\left(K_{\mathrm{S}}-[15]\right)_{0}$ in the range $0.3-0.5 \mathrm{mag}$. The variation in $\left(K_{\mathrm{s}}-[15]\right)_{0}$ leads to an uncertainty in $\dot{M}$ that could be up to a factor of 3 for $\dot{M} \sim$ $10^{-7} M_{\odot} / \mathrm{yr}$, which corresponds to most of the sources with large mass-loss (Fig. 12, see also Fig. 11a).

Alternatively, one could have also inferred $\dot{M}$ from $\left(K_{\mathrm{S}}-[7]\right)_{0}$ or from [7]-[15] colours. Deriving $\dot{M}$ from [7]-[15] (Alard et al. 2001) has the advantage that the latter quantity hardly depends on the extinction. However, this method is less sensitive since the variation in [7]-[15] is low, but $\left(K_{\mathrm{S}}-[7]\right)_{0}$ has low sensitivity for low mass-loss rates, but could be conveniently used for high mass-loss rates.

\subsection{Candidate young stellar objects}

In the current work, sources with an excess in the mid-infrared (see Fig. 13a) and relatively small near-infrared colours, $\left(J-K_{\mathrm{S}}\right)$ are identified as YSO candidates. The properties listed above are indicative of relatively nearby, less extincted sources, so that the mid-infrared excess has to arise from circumstellar dust in which the star may be embedded. All of these $15 \mu \mathrm{m}$ sources have $7 \mu \mathrm{m}$ counterparts, and the $7 \mu \mathrm{m}$ also shows an excess in the $K_{\mathrm{S}}-$ [7] colour (see Fig. 13b). There are two faint $7 \mu \mathrm{m}$ sources without $15 \mu \mathrm{m}$ counterparts. We include these in the list of candidate YSOs because they have GLIMPSE colours (Fig. 14a) consistent with those expected for YSOs.

In the past, the characteristic ISOGAL diagram, [15] vs. [7]-[15] (Fig. 10b) has been used by several authors (e.g. Felli et al. 2000, 2002; Schuller et al. 2006) to identify candidate YSOs. Felli et al. (2002) identify a simple criteria ([7]-[15] > $2.0)$ to qualify a source as a candidate YSO. In LN45 field, we find six sources that satisfy the Felli et al. (2002) colour criteria. However, one of them is a well known planetary nebula (ESO 134-7), as mentioned earlier. Most of the sources with

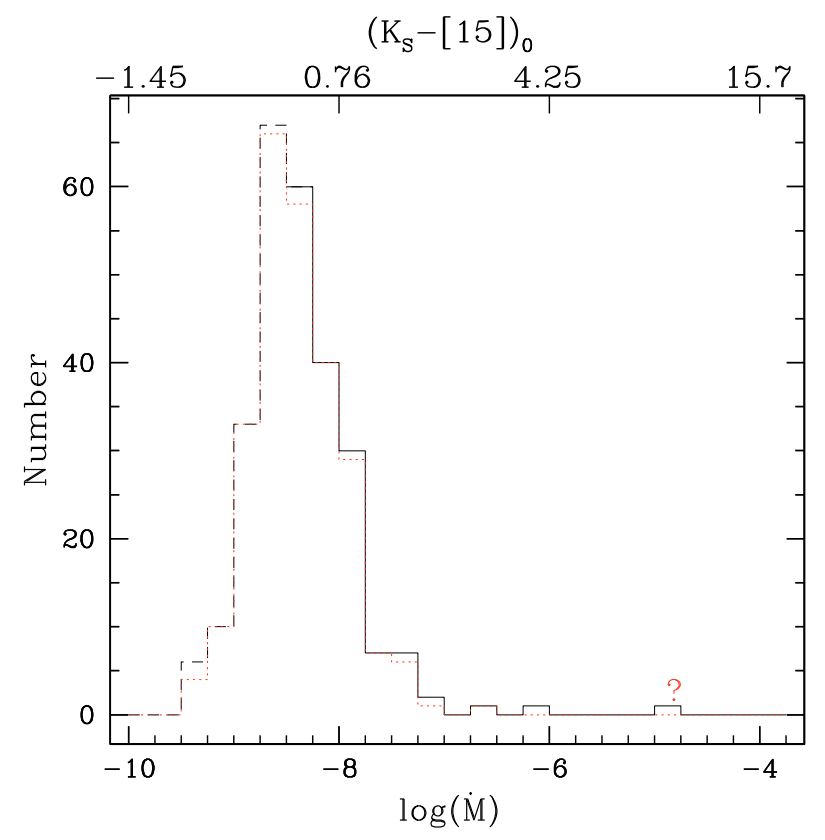

Fig. 12. Histogram of the mass-loss rates (bottom axis) determined from the $\left(K_{\mathrm{S}}-[15]\right)_{0}$ colours (top axis). The (red) dotted histogram is obtained after discarding sources with $J$ band missing in the GLIMPSE2MASS catalogue. Histogram for the mass-loss rates below the value of $\log \dot{M}=-8$ are represented by a dashed line. The contribution of source 729 with uncertain distance (see text) is marked by a question mark.

[7] $-[15]>2.0$ are relatively fainter than the limit required by Felli et al. (2002). This colour criterion is neither a sufficient nor a necessary condition as evinced by the large number of YSO candidates with relatively bluer colours. Schultheis et al. (2003) have shown that the ISOGAL [7]-[15] colour, by itself, is not sufficient to identify YSO candidates, and one requires additional data such as spectroscopic followup.

Figures 14a and $\mathrm{b}$ show the GLIMPSE CCD and CMD. In the GLIMPSE CCD, we have marked the area occupied by class II YSOs (Allen et al. 2004) by a rectangular box. Ten $15 \mu \mathrm{m}$ sources identified as YSOs occupy this region. Most of the other sources have colours consistent with class I types (i.e. with redder GLIMPSE colours). A few YSO candidates have relatively bluer GLIMPSE colours. These are sources with an excess in the $K_{\mathrm{S}}-$ [15], [7]-[15] colours and are relatively faint but still significant at $15 \mu \mathrm{m}$. Additional information would be needed to determine their true nature.

\section{Conclusion}

We have studied a large ISOGAL field towards the Galactic disc and established a standard mode of studying the large set of ISOGAL observations of the disc. We show that the stars in the red clump can be traced along the line of sight using the 2MASS $J$ and $K_{\mathrm{S}}$ data up to the start of the Scutum-Crux arm towards the $\ell=-45^{\circ}$ direction. We find that the locally accepted value of the extinction per unit distance, $c_{J}$, is sufficient to fit the red clump locus well up to $2.5 \mathrm{kpc}$ in this direction. However, beyond $2.5 \mathrm{kpc}, c_{J}$ varies with galactic latitude and increases with distance. We use the red clump locus to obtain the distance and extinction towards individual stars assuming them to be red giants and thus following the RGB/AGB isochrone. The distribution of stellar density rises as one hits the spiral arm at $4 \mathrm{kpc}$. The 2MASS data are not deep enough to detect the stars of the 

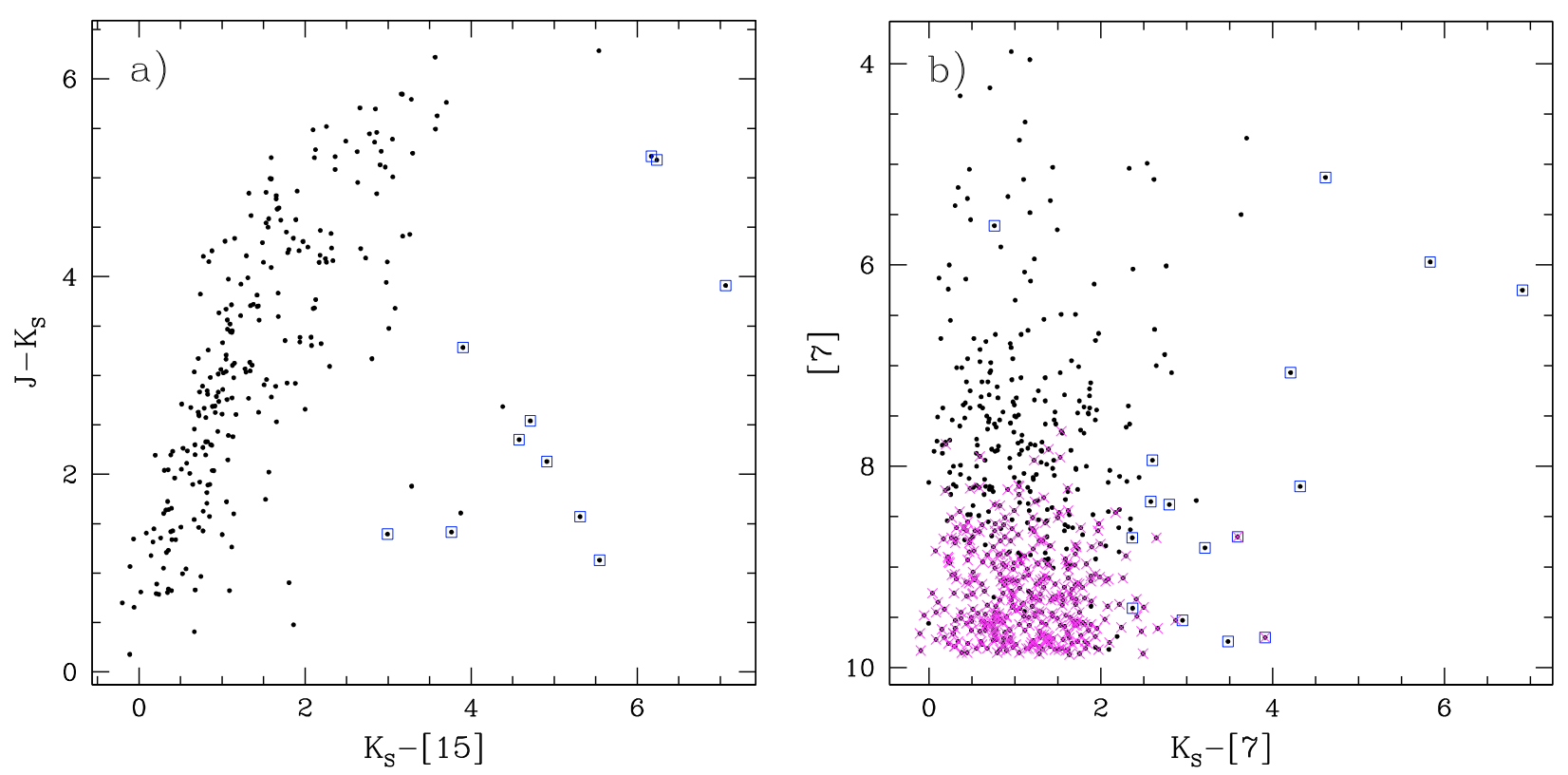

Fig. 13. a) $J-K_{\mathrm{S}}$ vs. $K_{\mathrm{S}}-$ [15] CCD with the YSO candidates plotted as open (blue) squares. b) [7] vs. $K_{\mathrm{S}}-$ [7] CMD. Magenta crosses are sources not detected at [15].
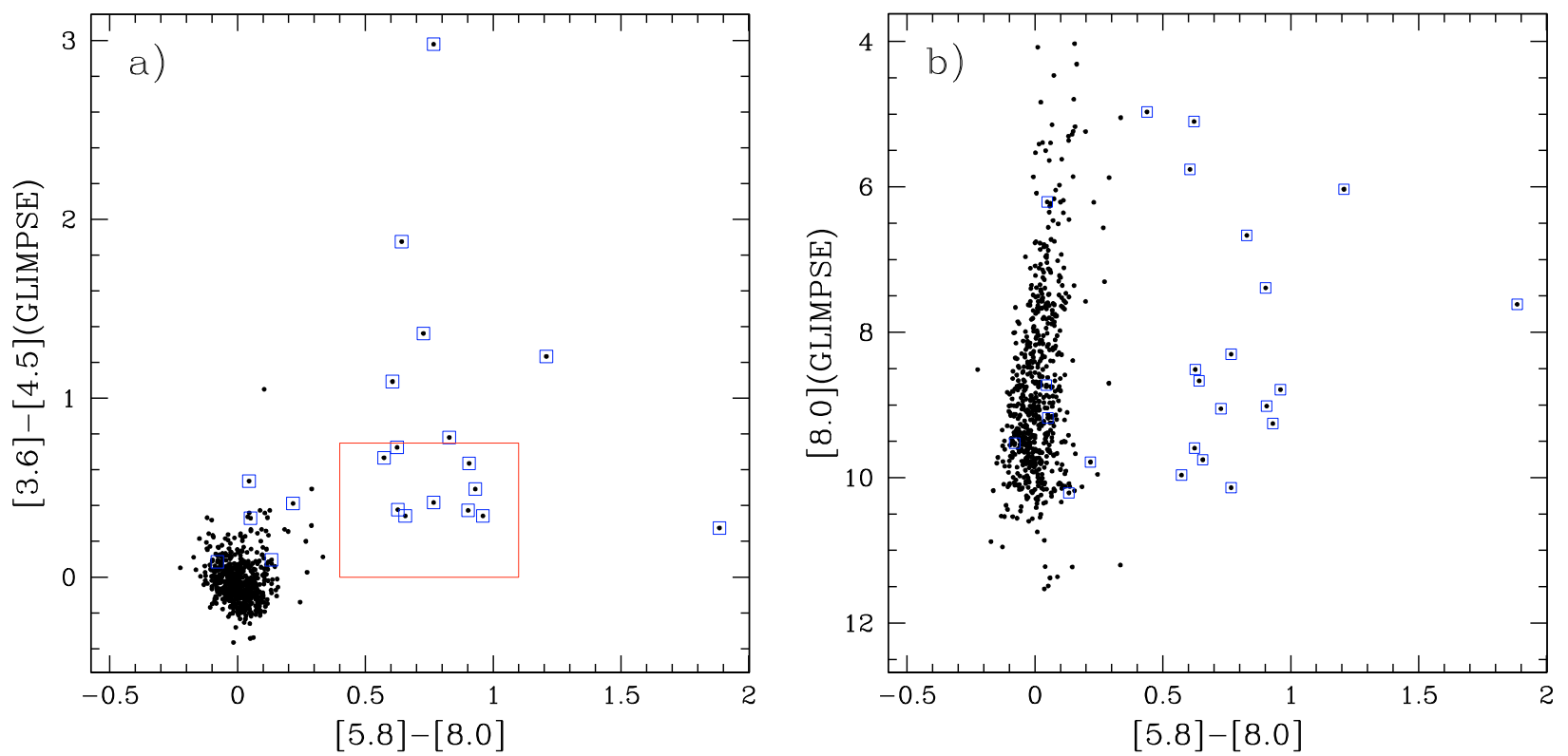

Fig. 14. a) GLIMPSE [5.8]-[8.0] vs. [3.6]-[4.5] CCD. The class II YSO region of Allen et al. (2004) is demarcated by a red box. b) GLIMPSE [5.8]-[8.0] vs. [8.0] CMD. YSO candidates are shown as open (blue) squares in both panels.

red clump at distances over $4 \mathrm{kpc}$ at the lowest galactic latitudes. We do see more luminous AGB stars to greater distances.

Most of the red giants brighter than the stars of the red clump are detected by ISOGAL at $7 \mu \mathrm{m}$. The $\left(K_{\mathrm{S}}-[15]\right)_{0}$ colour provides the mass-loss rates for the AGB stars. There are not many AGBs with a high mass-loss rate in this direction. From the mid infrared colour excess we identify a total of 22 YSO candidates in this field.

We provide a catalogue of the sources detected by ISOGAL with the estimated extinction and distance. We also tabulate the mass-loss rates for the several hundred red giants towards this field.

Acknowledgements. This work is based on observations made with the Spitzer Space Telescope, which is operated by the Jet Propulsion Laboratory, California
Institute of Technology, under a contract with NASA. We are grateful to the GLIMPSE project for providing access to the data. This publication makes use of data products from the Two Micron All Sky Survey, which is a joint project of the University of Massachusetts and the Infrared Processing and Analysis Center/California Institute of Technology, funded by the National Aeronautics and Space Administration and the National Science Foundation. This research made use of data products from the Midcourse Space Experiment. Processing of the data was funded by the Ballistic Missile Defense Organization with additional support from NASA Office of Space Science. This research made use of the SIMBAD database, operated at the CDS, Strasbourg, France. We acknowledge use of the TOPCAT software from Starlink and the VOPLOT virtual observatory software from VO-India (IUCAA) in this work. We thank the referee, Sean Carey, for constructive comments and criticisms that have improved the paper. S. Ganesh was supported by Marie-Curie EARA fellowship to work at the Institut d'Astrophysique de Paris. We are grateful to the Indo-French Astronomy Network for providing travel support to enable M. Schultheis to visit the PRL. Work at the Physical Research Laboratory is supported by the Department of Space, Govt. of India. 


\section{Appendix A: Flag definition for ISOGAL-GLIMPSE association: readme file}

rQ $=$ radius of association for $10 \%$ chance of false association rQ $=3.8$ for new GLIMPSE catalogue with 4601 IRAC4 (i4) sources for LN45 $r 1=2.3^{\prime}$ ' and $\mathrm{r} 2=1.5$ for LN45 field m7 - LW2 detection; m15 - LW3 detection; m81 - nearest GLIMPSE source; m82 - next (second) nearest GLIMPSE source

Flag $=9:$ Saturated in GLIMPSE i4:

ISOGAL (m7) brighter than 4 and no source in GLIMPSE i4

Flag = 8: Not at saturation level but no GLIMPSE - could be due to extendedness

ISOGAL ( $\mathrm{m} 7 \& \mathrm{~m} 15)$ present and ( $\mathrm{m} 7$ fainter than 4 and $\mathrm{m} 15$ brighter than 5 ) and no source in GLIMPSE i4

Flag = 7: Not at saturation level and GLIMPSE source in $r>r \theta$ and $r<4.5$, ,

Flag $=5:$ very secure associations

(separation $<1.5 \& \&$ abs $(m 7-i 4)<0.3)$

i.e. $r<r 2$ and $|\mathrm{m} 7-\mathrm{m} 81|<0.3$ and no other GLIMPSE source within $r 0$

Flag $=4:$ secure association

$(1.5<$ separation \&\& separation $<2.3$ \&\& abs $(m 7-i 4)<0.5)||$

(separation $<1.5 \& \&$ abs $(m 7-i 4)<0.5 \& \&$ abs $(m 7-i 4)>0.3)$

$4.1 \mathrm{r} 2<\mathrm{r}<\mathrm{r} 1$ and $|\mathrm{m} 7-\mathrm{m} 81|<0.5$ and no other GLIMPSE source within r0

$4.2 \mathrm{r}<\mathrm{r} 2$ and $0.3<|\mathrm{m} 7-\mathrm{m} 81|<0.5$ and no other GLIMPSE source within $\mathrm{r} \theta$

$4.3 \mathrm{r}<\mathrm{r} 2$ and $|\mathrm{m} 7-\mathrm{m} 81|<0.3$ and $\mathrm{m} 82>\mathrm{m} 7+1$

(i.e. second GLIMPSE source fainter by atleast 1mag)

Flag $=3:$ probable association

(separation $>2.3 \& \&$ abs $(m 7-i 4)<0.5)||$

(separation $<2.3 \& \&$ abs $(m 7-i 4)<1.2 \& \&$ abs $(m 7-i 4)>0.5)||$

(separation $<2.3 \& \& \mathrm{~m} 7>20$ )

$3.1 \mathrm{r} 1<\mathrm{r}<\mathrm{r} \theta$ with $|\mathrm{m} 7-\mathrm{m} 81|<0.5$ and no other GLIMPSE source within $\mathrm{r} \theta$

$3.2 \mathrm{r}<\mathrm{r} 1$ with $0.5<|\mathrm{m} 7-\mathrm{m} 81|<1.2$ and no other GLIMPSE source within $\mathrm{r} \theta$

$3.3 \mathrm{r}<\mathrm{r} 1$ with $15 \mathrm{micron}$ detection only and no other GLIMPSE source within r0

$3.4 \mathrm{r}<\mathrm{r} 1$ for second neighbour with $|\mathrm{m} 7-\mathrm{m} 82|<0.5$ and $|\mathrm{m} 7-\mathrm{m} 81|>0.5$

- keep the second neighbour as association in this case

Flag $=2:$ possible association

(separation $<3.8$ \&\& separation $>2.3 \& \&$ abs $(m 7-i 4)<1.2 \& \&$ abs $(m 7-i 4)>0.5)||$

(separation $<2.3 \& \&$ abs $(m 7-i 4)>1.2 \& \&$ abs $(m 7-i 4)<2.2) \|$

(separation $>2.3 \& \& \mathrm{~m} 7>20$ )

$2.1 \mathrm{r} 1<\mathrm{r}<\mathrm{r} \theta$ with $0.5<|\mathrm{m} 7-\mathrm{m} 81|<1.2$ and no other GLIMPSE

$2.2 \mathrm{r}<\mathrm{r} 1$ with $1.2<|\mathrm{m} 7-\mathrm{m} 81|<2.2$ and no other GLIMPSE

$2.3 \mathrm{r} 1<\mathrm{r}<\mathrm{r} \theta$ with $15 \mathrm{micron}$ only and no other GLIMPSE within $\mathrm{r} \theta$

$2.4 \mathrm{r} 1<\mathrm{r}<\mathrm{r} 0$ for second neighbour with $|\mathrm{m} 7-\mathrm{m} 82|<1$ and $|\mathrm{m} 7-\mathrm{m} 81|>1.5$

- keep the second neighbour as association in this case

Flag = 1: hesitation to reject

(separation $<2.3 \& \&$ abs $(m 7-i 4)>2.2 \& \&$ abs $(m 7-i 4)<2 \theta) \quad \mid$

(separation $>2.3 \& \&$ separation $<3.8 \& \&$ abs $(m 7-i 4)>1.2)||$

(NULL_separation $\& \& \mathrm{~m} 7<20 \& \& \mathrm{~m} 15<20$ )

$1.1 \mathrm{r}<\mathrm{r} 1$ and $|\mathrm{m} 7-\mathrm{m} 81|>2.2$

$1.2 \mathrm{r} 1<\mathrm{r}<\mathrm{r} 2$ and $|\mathrm{m} 7-\mathrm{m} 81|>1.2$

- could be recovered with further info eg 24 micron...

e.g. $7 \& 15$ only and no GLIMPSE within $r \theta$

or 7 with $|\mathrm{m} 7-\mathrm{m} 81|>2.2$ and $\mathrm{r}<\mathrm{r} \theta$ and no other GLIMPSE

Flag $=0:$ rejects - separate file

NULL_separation $\& \&(\mathrm{~m} 7>20|| \mathrm{m} 15>20)$

NOTES: - based on the GLIMPSE source quality flag: if the SQF bit 14 is set (i.e. 8192), then degrade the ultimate ISOGAL+GLIMPSE quality flag by 1.

- if there is also a detection at LW3 then increase the flag value by 1

- this is for sources with otherwise flags of 2 and 1 based on $m 7$ values. 


\section{Appendix B: Multi-wavelength catalogue for LN45 field}

Table B.1. An extract of the multi-wavelength catalogue with the mass-loss rate, distance, extinction values, and remarks on individual sources. The complete catalogue will be made available at the CDS.

\begin{tabular}{|c|c|c|c|c|}
\hline Column name & Units & Source 1 & Source 2 & Source 3 \\
\hline id & number & 2 & 14 & 187 \\
\hline RA & deg & 217.5421 & 217.6252 & 217.8831 \\
\hline Dec & deg & -60.0549 & -60.0626 & -60.113 \\
\hline ISOGAL-DENIS PSC1 & name & PJ143010.1-600317 & PJ143030.0-600345 & PJ143131.9-600646 \\
\hline DENIS $I$ & mag & 12.35 & 99.99 & 13.96 \\
\hline DENIS $J$ & mag & 10.69 & 99.99 & 8.62 \\
\hline DENIS $K_{\mathrm{S}}$ & mag & 9.62 & 99.99 & 5.55 \\
\hline 2MASS $J$ & mag & 10.718 & 11.55 & 8.565 \\
\hline 2MASS $H$ & mag & 9.837 & 9.05 & 6.884 \\
\hline $2 \mathrm{MASS} K_{\mathrm{S}}$ & mag & 9.626 & 7.878 & 6.035 \\
\hline IRAC1 & mag & 9.479 & 7.106 & 99.999 \\
\hline IRAC2 & mag & 9.6 & 7.062 & 99.999 \\
\hline IRAC3 & mag & 9.435 & 6.787 & 5.422 \\
\hline IRAC4 & mag & 9.39 & 6.724 & 5.277 \\
\hline LW2 & mag & 9.16 & 6.54 & 5.55 \\
\hline LW3 & mag & 99.99 & 5.78 & 4.38 \\
\hline MSX B1 & Jy & & $-1.412 \mathrm{e} 1$ & $-1.223 \mathrm{e}+1$ \\
\hline MSX B2 & Jy & & $-7.623 \mathrm{e}+0$ & $2.690 \mathrm{e}+0$ \\
\hline MSX A & Jy & & $9.725 \mathrm{e}-2$ & $5.268 \mathrm{e}-1$ \\
\hline MSX C & Jy & & $-7.769 \mathrm{e}-1$ & $4.335 \mathrm{e}-1$ \\
\hline MSX D & Jy & & $-5.570 \mathrm{e}-1$ & $3.338 \mathrm{e}-1$ \\
\hline MSX E & Jy & & $-1.772 \mathrm{e}+0$ & $4.387 \mathrm{e}-1$ \\
\hline ISOGAL-GLIMPSE association & flag & 5 & 4 & 5 \\
\hline Source type & text & RGB & RGB & RGB \\
\hline Distance & $\mathrm{pc}$ & 2369 & 6512 & 4000 \\
\hline Extinction & $\operatorname{mag} A_{\mathrm{V}}$ & 1.48 & 14.02 & 6.56 \\
\hline Absolute magnitude $\mathrm{M} K_{\mathrm{S}}$ & mag & -2.37 & -7.43 & -7.55 \\
\hline $\log$ Mass-loss rate $\log (\dot{M})$ & $\log \left(M_{\odot} / \mathrm{yr}\right)$ & - & -7.69 & -7.66 \\
\hline Remarks & text & & AGB & IRAC1, 2 saturated \\
\hline
\end{tabular}


Appendix $C: J-K_{S}$ vs. $J$ colour-magnitude diagrams selected by survey wavelengths
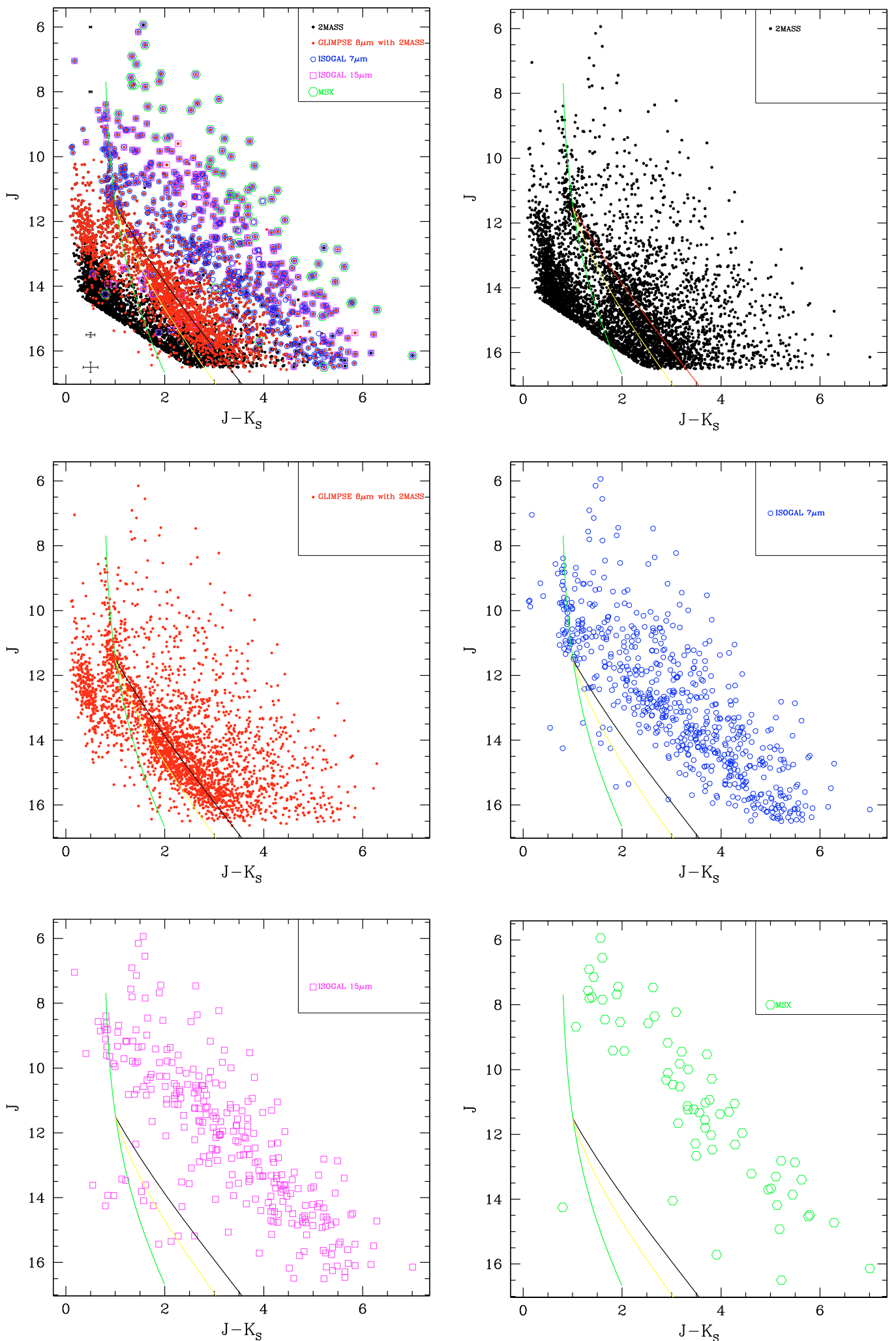

Fig. C.1. $J-K_{\mathrm{S}}$ vs. $J$ colour-magnitude diagrams with the different survey detections displayed separately. The solid lines are the red clump loci with standard $c_{J}$ (i.e. local value of $c_{J}$ being used throughout) displayed in green colour; locus towards $b=0.5$ in yellow; locus towards $b=0.0$ in black. 
Appendix D: Colour-colour diagrams to estimate mid-infrared extinction coefficients
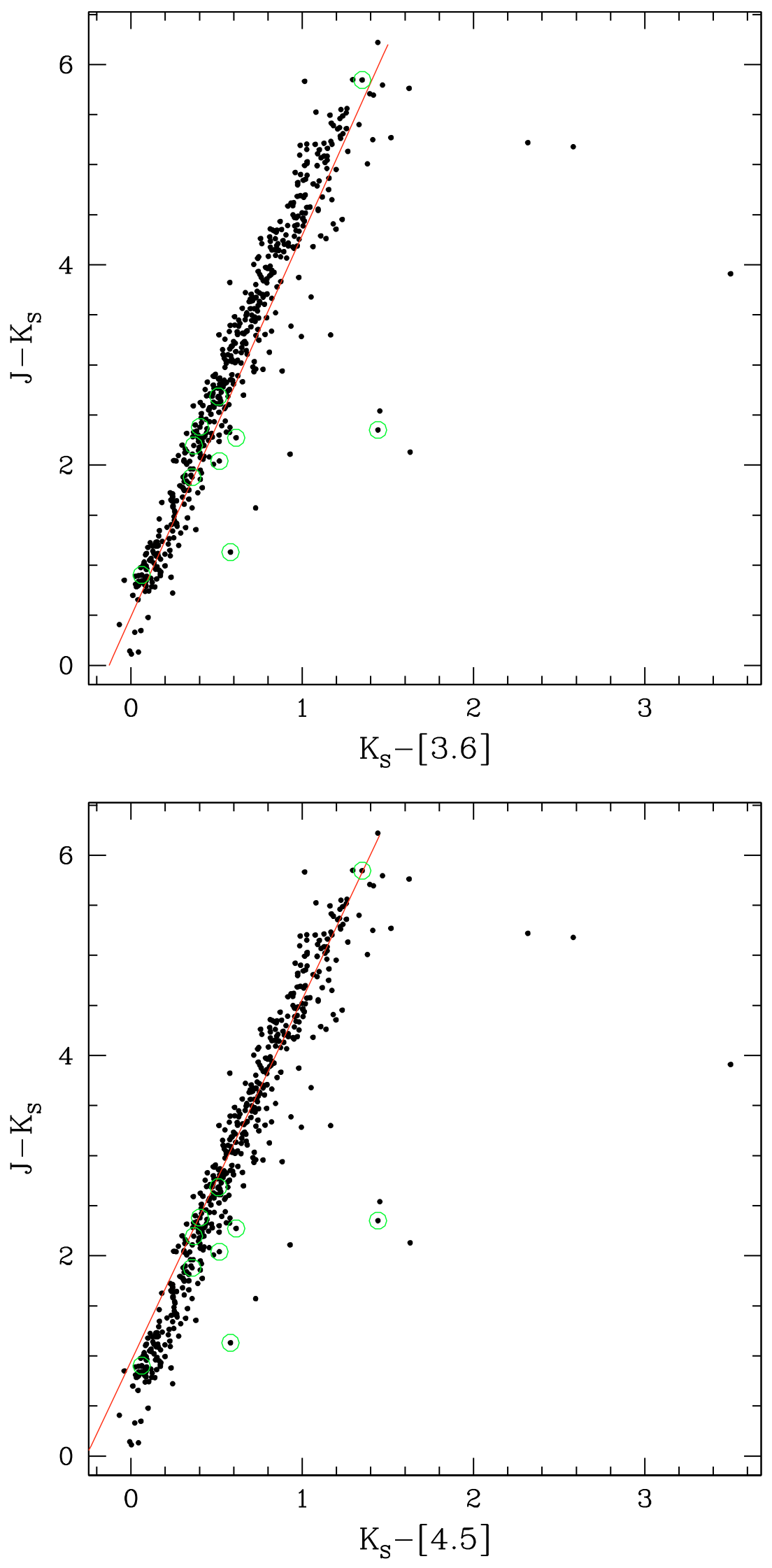

Fig. D.1. $J-K_{\mathrm{S}}$ vs. $K_{\mathrm{S}}-[3.6]$ colour-colour diagram. Sources detected only at [15] are marked with an additional green open circle. The fit used to derive the extinction coefficient at $3.6 \mu \mathrm{m}$ is shown as a solid red line.
Fig. D.2. $J-K_{\mathrm{S}}$ vs. $K_{\mathrm{S}}-[4.5]$ colour-colour diagram. Sources detected only at [15] are marked with an additional green open circle. The fit used to derive the extinction coefficient at $4.5 \mu \mathrm{m}$ is shown as a solid red line. 


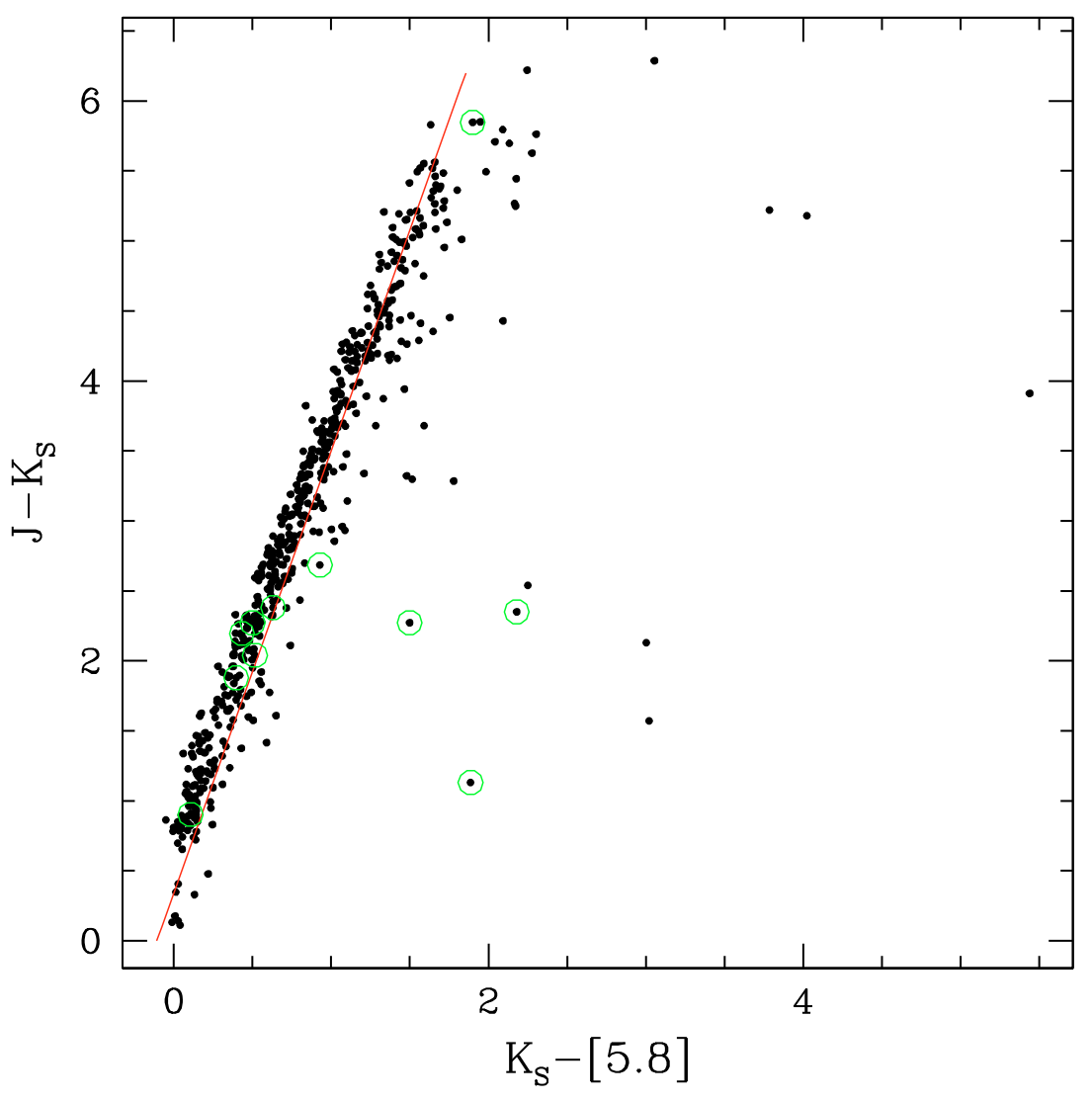

Fig. D.3. $J-K_{\mathrm{S}}$ vs. $K_{\mathrm{S}}-[5.8]$ colour-colour diagram. Sources detected only at [15] are marked with an additional green open circle. The fit used to derive the extinction coefficient at $5.8 \mu \mathrm{m}$ is shown as a solid red line.

\section{Appendix E: $J-K_{S}$ vs. $J$ colour-magnitude diagrams for various ranges in $b$}

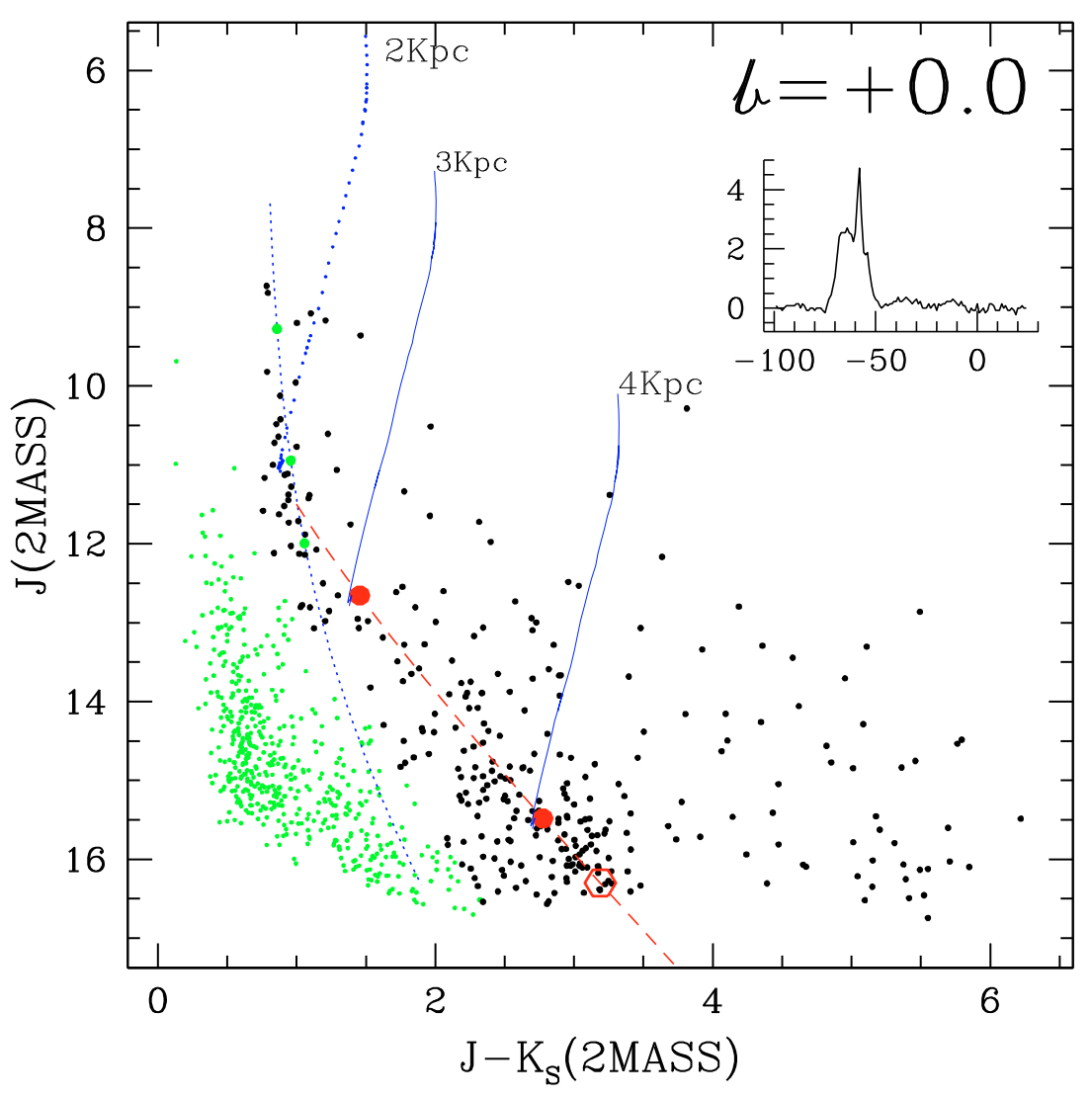

Fig. E.1. $J-K_{\mathrm{S}}$ vs. $J \mathrm{CMD}$ for the latitude ranges corresponding to the $\mathrm{CO}$ cell latitude limits $\left(b_{\text {low }}=\right.$ $-0.0625, b_{\text {high }}=0.0625$ ) centred at $b=0.0$. Also shown are the RGB/AGB isochrones at various distances as labelled in the figure. We use $A_{\mathrm{V}}=6.0\left(A_{J}-\right.$ $A_{K_{\mathrm{S}}}$ ) (see Table 4). Large open hexagons show the points listed in Table 3 . The corresponding $\mathrm{CO}$ spectrum is shown as an inset with the velocity scale (in $\mathrm{km} \mathrm{s}^{-1}$ ) marked on the $x$ axis. 

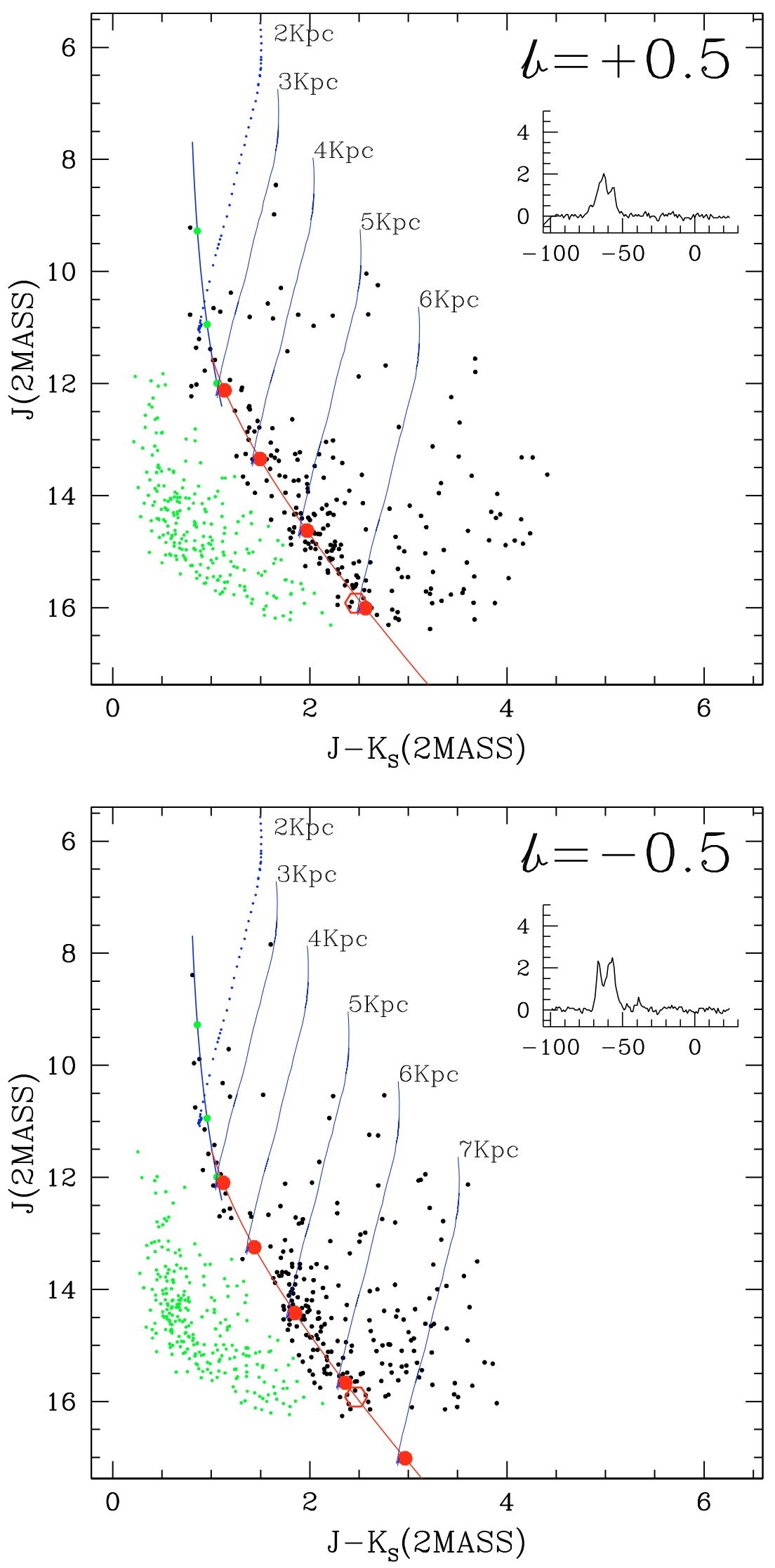

Fig. E.2. $J-K_{\mathrm{S}}$ vs. $J \mathrm{CMD}$ for the latitude ranges corresponding to the $\mathrm{CO}$ cell latitude limits $\left(b_{\text {low }}=0.4375\right.$, $b_{\text {high }}=0.5$ ) centred at $b=0.5$. Rest of the details are as in Fig. E.1.

Fig. E.3. $J-K_{\mathrm{S}}$ vs. $J \mathrm{CMD}$ for the latitude ranges corresponding to the $\mathrm{CO}$ cell latitude limits $\left(b_{\text {low }}=-0.5\right.$, $\left.b_{\text {high }}=-0.4375\right)$ centred at $b=-0.5$. Rest of the details are as in Fig. E.1. 

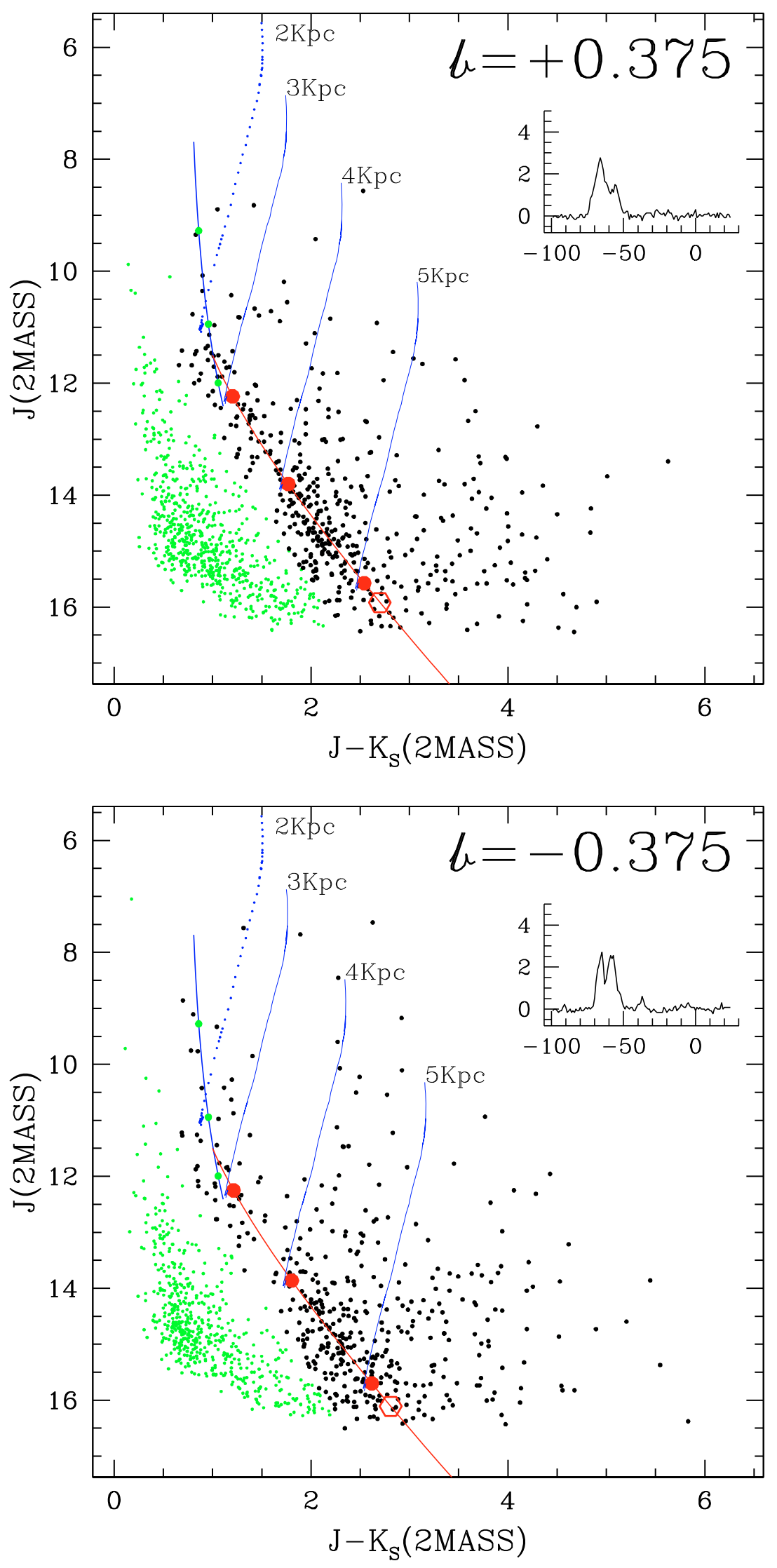

Fig. E.4. $J-K_{\mathrm{S}}$ vs. $J \mathrm{CMD}$ for the latitude ranges corresponding to the $\mathrm{CO}$ cell latitude limits $\left(b_{\text {low }}=0.3125\right.$, $b_{\text {high }}=0.4375$ ) centred at $b=0.375$. Rest of the details are as in Fig. E.1.

Fig. E.5. $J-K_{\mathrm{S}}$ vs. $J$ CMD for the latitude ranges corresponding to the $\mathrm{CO}$ cell latitude limits $\left(b_{\text {low }}=\right.$ $-0.4375, b_{\text {high }}=-0.3125$ ) centred at $b=-0.375$. Rest of the details are as in Fig. E.1. 

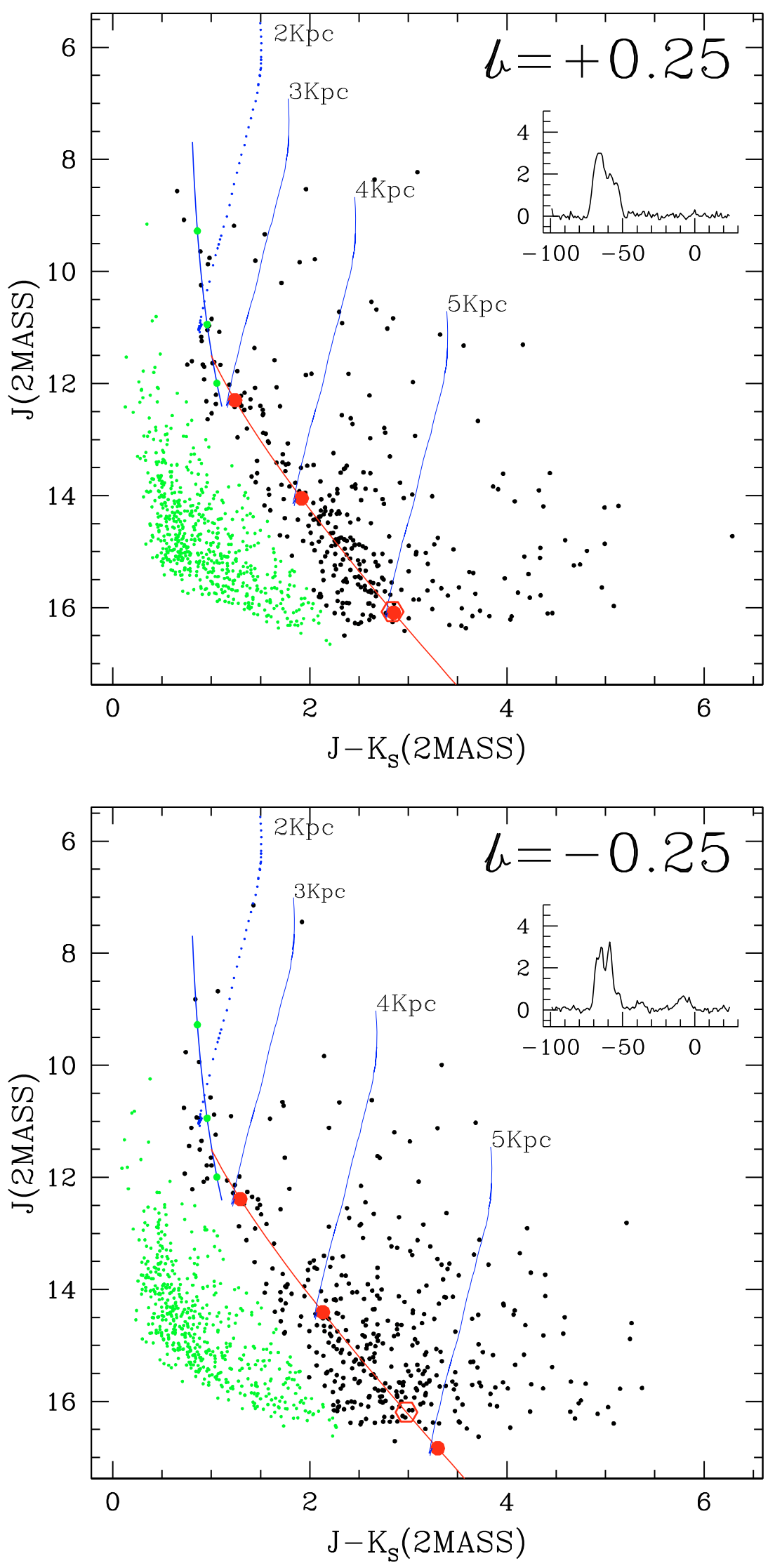

Fig. E.6. $J-K_{\mathrm{S}}$ vs. $J \mathrm{CMD}$ for the latitude ranges corresponding to the $\mathrm{CO}$ cell latitude limits $\left(b_{\text {low }}=0.1875\right.$, $\left.b_{\text {high }}=0.3125\right)$ centred at $b=0.25$. Rest of the details are as in Fig. E.1.

Fig. E.7. $J-K_{\mathrm{S}}$ vs. $J$ CMD for the latitude ranges corresponding to the $\mathrm{CO}$ cell latitude limits $\left(b_{\text {low }}=\right.$ $\left.-0.3125, b_{\text {high }}=-0.1875\right)$ centred at $b=-0.25$. Rest of the details are as in Fig. E.1. 

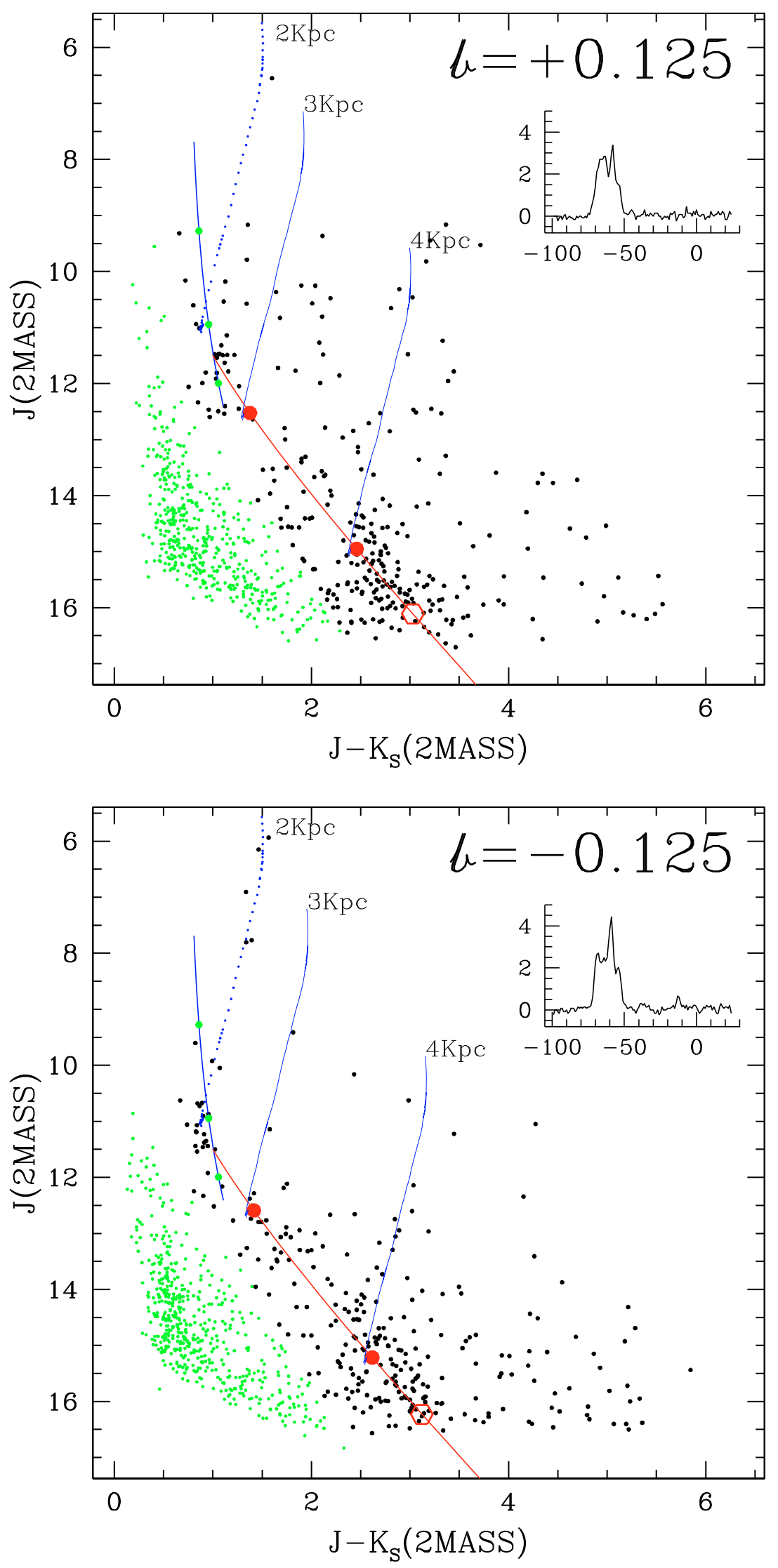

Fig. E.8. $J-K_{\mathrm{S}}$ vs. $J \mathrm{CMD}$ for the latitude ranges corresponding to the $\mathrm{CO}$ cell latitude limits $\left(b_{\text {low }}=0.0625\right.$, $b_{\text {high }}=0.1875$ ) centred at $b=0.125$. Rest of the details are as in Fig. E.1.

Fig. E.9. $J-K_{\mathrm{S}}$ vs. $J$ CMD for the latitude ranges corresponding to the $\mathrm{CO}$ cell latitude limits $\left(b_{\text {low }}=\right.$ $\left.-0.1875, b_{\text {high }}=-0.0625\right)$ centred at $b=-0.125$. Rest of the details are as in Fig. E.1. 


\section{References}

Alard, C., Blommaert, J. A. D. L., Cesarsky, C., et al. 2001, ApJ, 552, 289

Alcock, C., Allsman, R. A., Alves, D., et al. 1997, ApJ, 479, 119

Allen, L. E., Calvet, N., D’Alessio, P., et al. 2004, ApJS, 154, 363

Benjamin, R. A., Churchwell, E., Babler, B. L., et al. 2003, PASP, 115, 953

Benjamin, R. A., Churchwell, E., Babler, B. L., et al. 2005, ApJ, 630, L149

Bertelli, G., Bressan, A., Chiosi, C., Fagotto, F., \& Nasi, E. 1994, A\&AS, 106, 275

Blommaert, J. A. D. L., Siebenmorgen, R., Coulais, A., et al. 2003, The ISO Handbook, Vol. II - CAM, The ISO Camera Version 2.0 (June), ed. T. G. Mueller, J. A. D. L. Blommaert, \& P. Garcia-Lario, ESA SP-1262, European Space Agency

Carey, S. J., Noriega-Crespo, A., Price, S. D., et al. 2005, in BAAS, 37, 1252

Cesarsky, C. J., Abergel, A., Agnese, P., et al. 1996, A\&A, 315, L32

Dame, T. M., Ungerechts, H., Cohen, R. S., et al. 1987, ApJ, 322, 706

Drimmel, R., Cabrera-Lavers, A., \& López-Corredoira, M. 2003, A\&A, 409, 205

Dutra, C. M., Santiago, B. X., Bica, E. L. D., \& Barbuy, B. 2003, MNRAS, 338, 253

Egan, M. P., Price, S. D., Kraemer, K. E., et al. 2003, Air Force Research Laboratory Technical Report AFRL-VS-TR-2003-1589, see VizieR Online Data Catalog, 5114, 0

Felli, M., Comoretto, G., Testi, L., Omont, A., \& Schuller, F. 2000, A\&A, 362, 199

Felli, M., Testi, L., Schuller, F., \& Omont, A. 2002, A\&A, 392, 971

Flaherty, K. M., Pipher, J. L., Megeath, S. T., et al. 2007, ApJ, 663, 1069

Frogel, J. A., \& Whitford, A. E. 1987, ApJ, 320, 199

Glass, I. S. 1999, Handbook of Infrared Astronomy, Highlights of Astronomy

Glass, I. S., Ganesh, S., Alard, C., et al. 1999, MNRAS, 308, 127

Groenewegen, M. A. T. 2006, A\&A, 448, 181

Indebetouw, R., Mathis, J. S., Babler, B. L., et al. 2005, ApJ, 619, 931

Jiang, B. W., Gao, J., Omont, A., Schuller, F., \& Simon, G. 2006, A\&A, 446, 551

Jiang, B. W., Omont, A., Ganesh, S., Simon, G., \& Schuller, F. 2003, A\&A, 400, 903

Kerber, F., Mignani, R. P., Guglielmetti, F., \& Wicenec, A. 2003, A\&A, 408, 1029
Lang, K. R. 1999, Astrophysical formulae (New York: Springer), ed. K. R. Lang, Astronomy and astrophysics library, ISSN0941-7834

Le Bertre, T., \& Winters, J. M. 1998, A\&A, 334, 173

López-Corredoira, M., Cabrera-Lavers, A., Garzón, F., \& Hammersley, P. L. 2002, A\&A, 394, 883

Marshall, D. J., Robin, A. C., Reylé, C., Schultheis, M., \& Picaud, S. 2006, A\&A, 453, 635

McCallon, H. L., Fowler, J. W., Laher, R. R., Masci, F. J., \& Moshir, M. 2007, PASP, 119, 1308

Morgan, D. H., Parker, Q. A., \& Cohen, M. 2003, MNRAS, 346, 719

Neckel, T., Klare, G., \& Sarcander, M. 1980, A\&AS, 42, 251

Ojha, D. K., Omont, A., Schuller, F., et al. 2003, A\&A, 403, 141

Omont, A., Ganesh, S., Alard, C., et al. 1999, A\&A, 348, 755

Omont, A., Gilmore, G. F., Alard, C., et al. 2003, A\&A, 403, 975

Ott, S., Gastaud, R., Guest, S., et al. 1999, in Astronomical Data Analysis Software and Systems VIII, ed. D. M. Mehringer, R. L. Plante, \& D. A. Roberts, ASP Conf. Ser., 172, 7

Palanque-Delabrouille, N., Afonso, C., Albert, J. N., et al. 1998, A\&A, 332, 1

Perault, M., Omont, A., Simon, G., et al. 1996, A\&A, 315, L165

Price, S. D., Egan, M. P., Carey, S. J., Mizuno, D. R., \& Kuchar, T. A. 2001, AJ, 121,2819

Robin, A. C., Reylé, C., Derrière, S., \& Picaud, S. 2003, A\&A, 409, 523

Román-Zúñiga, C. G., Lada, C. J., Muench, A., \& Alves, J. F. 2007, ApJ, 664, 357

Russeil, D. 2003, A\&A, 397, 133

Schuller, F. 2002, Ph.D. Thesis, Institut d'Astrophysique de Paris, Universite Pierre et Marie Curie - Paris VI

Schuller, F., Ganesh, S., Messineo, M., et al. 2003, A\&A, 403, 955

Schuller, F., Omont, A., Glass, I. S., et al. 2006, A\&A, 453, 535

Schultheis, M., Ganesh, S., Glass, I. S., et al. 2000, A\&A, 362, 215

Schultheis, M., Ganesh, S., Simon, G., et al. 1999, A\&A, 349, L69

Schultheis, M., Lançon, A., Omont, A., Schuller, F., \& Ojha, D. K. 2003, A\&A, 405,531

Wainscoat, R. J., Cohen, M., Volk, K., Walker, H. J., \& Schwartz, D. E. 1992, ApJS, 83, 111

Werner, M. W., Roellig, T. L., Low, F. J., et al. 2004, ApJS, 154, 1

Whitelock, P., Menzies, J., Feast, M., et al. 1994, MNRAS, 267, 711 